Florida International University FIU Digital Commons

5-14-2015

\title{
African American and Afro-Caribbean American Men's Prostate Health Knowledge and Beliefs
}

Cora Yoose

Florida International University, cyoos001@fiu.edu

DOI: $10.25148 /$ etd.FIDC000169

Follow this and additional works at: https://digitalcommons.fiu.edu/etd

Part of the Nursing Commons

\section{Recommended Citation}

Yoose, Cora, "African American and Afro-Caribbean American Men's Prostate Health Knowledge and Beliefs" (2015). FIU Electronic Theses and Dissertations. 2272.

https://digitalcommons.fiu.edu/etd/2272

This work is brought to you for free and open access by the University Graduate School at FIU Digital Commons. It has been accepted for inclusion in FIU Electronic Theses and Dissertations by an authorized administrator of FIU Digital Commons. For more information, please contact dcc@fiu.edu. 


\section{FLORIDA INTERNATIONAL UNIVERSITY}

Miami, Florida

\section{AFRICAN AMERICAN AND AFRO-CARIBBEAN AMERICAN MEN'S PROSTATE}

HEALTH KNOWLEDGE AND BELIEFS

A dissertation submitted in partial fulfillment of the

requirements for the degree of

DOCTOR OF PHILOSOPHY

in

NURSING

by

Cora Yoose

2015 
To: Dean Ora Strickland

College of Nursing and Health Sciences

This dissertation, written by Cora Yoose, and entitled African American and AfroCaribbean American Men's Prostate Health Knowledge and Beliefs, having been approved in respect to style and intellectual content, is referred to you for judgment.

We have read this dissertation and recommend that it be approved.

Eric Fenkl

Sheldon Fields

Jean Hannan

Timothy Page

Amy Paul-Ward

Date of Defense: May 14, 2015

Ellen Brown, Major Professor

The dissertation of Cora Yoose is approved.

Dean Ora Strickland

College of Nursing and Health Sciences

Dean Lakshmi Reddi

University Graduate School

Florida International University, 2015 
(C) Copyright 2015 by Cora Yoose

All rights reserved. 


\section{ACKNOWLEDGMENTS}

I wish to thank my chair, Dr. Ellen Brown, and the other members of my committee throughout this process: Dr. Eric Fenkl, Dr. Sheldon Fields, Dr. Jean Hannan, Dr. Timothy Page, Dr. Carol (Pat) Patsdaughter, and Dr. Amy Paul-Ward. I would like to convey my gratitude to Dr. Yvonne Parchment, Dr. Carmen Caicedo, Dr. Bruno Da Costa, Dr. Jianbin (Vincent) Zhu, Dr. Glenn Hutchinson, Dr. Anthony Miyazaki, Dr. Suha Ballout, Dr. Nisarg Karia, Dr. Linda Taylor, Corey Ginsberg, Christopher Ponce, Karen Iglesias, Stephanie Garcia, Stefanie Moore, and Muhammad Abdul Baker Chowdhury. I would also like to thank church leaders and members for their support and time coordinating with me. Finally, I would like to thank my friends, my family (especially my husband and mother-in-law), and God. 
ABSTRACT OF THE DISSERTATION

AFRICAN AMERICAN AND AFRO-CARIBBEAN AMERICAN MEN'S PROSTATE HEALTH KNOWLEDGE AND BELIEFS

\author{
by \\ Cora Yoose \\ Florida International University, 2015 \\ Miami, Florida

\section{Professor Ellen Brown, Major Professor}

Approximately one in every seven American men will be diagnosed with prostate cancer during his lifetime. Men of African descent have higher incidence and mortality rates than others. Prostate cancer screening is important because the five-year survival rate is only 31\% post-metastasis. The purpose of this study was to assess the likelihood of action for and factors influencing choice regarding prostate screenings. It was also to determine if a relationship existed between ethnicity (African American and AfroCaribbean American men) and knowledge of prostate health, self-efficacy, perceived barriers to and belief regarding prostate screening. Data collection methods included a focus group ( $n=8)$ among African American and Afro-Caribbean American men $(M=53.8,10.3)$ and self-administered surveys $(n=113)$ among African American $(n=49,45.4 \%)$ and Afro-Caribbean American $(n=38,35.2 \%)$ men $(M=59.5,16.4)$ from churches in South Florida using convenience sampling and the Health Belief Model (HBM) as a framework. Knowledge was assessed using a combined version of the Knowledge and Practice of Prostate Health Questionnaire and Prostate Cancer Screening Education (PROCASE) Knowledge Index. Self-efficacy was measured as decisional 
conflict reported from the Low Literacy Decisional Conflict Scale. Barriers were identified from a Perceived Barriers Survey. Beliefs were measured as spiritual wellbeing and evaluated using the Functional Assessment of Chronic Illness TherapySpiritual Well-Being, a modified version for non-illness (FACIT-Sp Non-Illness). Almost half of African American (47.9\%) and nearly a third (29\%) of Afro-Caribbean American participants were unaware of participation or did not participate in prostatespecific antigen (PSA) testing. Findings indicated prostate screening concerns, inadequate knowledge, past negative experiences, and cost may contribute to low prostate screening rates. Both ethnicities did not differ in knowledge of prostate health or selfefficacy for making an informed decision regarding prostate screening. Potential targets for outreach efforts among these ethnic groups could include faith-based medical partnerships to diminish health disparities. Future intervention studies would benefit from a focus on diverse cultures and ethnicities in different settings and culturally appropriate strategies for nurses and other health professionals to use when assisting patients with informed decision making regarding prostate cancer screening. 


\section{TABLE OF CONTENTS}

CHAPTER

PAGE

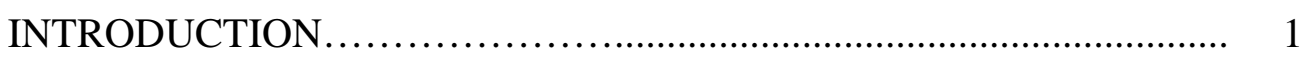

Significance of Prostate Cancer.................................................... 1

Significance of Culture......................................................... 1

Study Purpose........................................................................ 7

Research Questions.............................................................. 8

Conceptual Framework............................................................... 8

Study Variables for the Framework................................... 10

II

REVIEW OF THE LITERATURE..................................................... 12

Prevalence and Risk for Men of African Descent......................... 13

Screening............................................................................... 15

Screening Properties......................................................... 15

Why Screening is Important............................................. 17

Screening Controversy.................................................... 19

Perceived Barriers to Screenings.......................................... 24

Culture.............................................................................. 29

Importance of Culture in Research.................................... 29

Culturally Appropriate Strategies....................................... 31

Prostate Cancer Cost................................................................... 33

Methodological Choices......................................................... 37

Qualitative: Focus Group................................................ 37

Quantitative: Survey...................................................... 37

III METHODOLOGY ..................................................................... 41

Setting and Sample............................................................. 41

Procedures........................................................................... 43

Data Collection..................................................................... 44

Focus Group.............................................................. 44

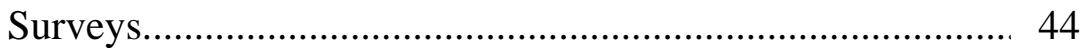

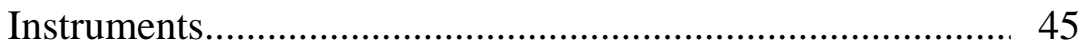

Data Management and Analysis................................................ 50

Human Participants.............................................................. 54

Risk to Human Participants.................................................. 54

Potential Benefits to Human Participants and Others......... 54

Adequacy of Protection against Risks................................. 55

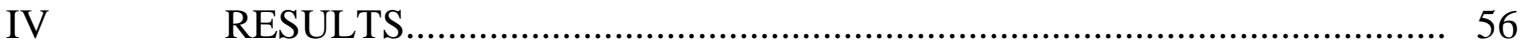

Sample of Focus Group Participants........................................... 56

Focus Group Questionnaire Packet.......................................... 56

Sample of Survey Participants.................................................. 57 
Prostate Knowledge.................................................................. 62

Decisional Conflict.......................................................................... 63

Barriers............................................................................. 65

Beliefs............................................................................. 66

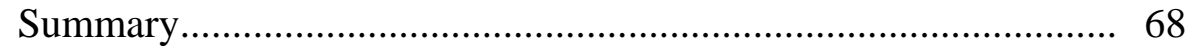

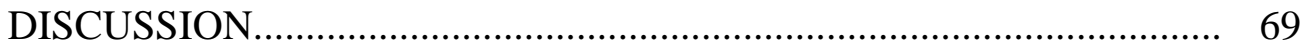

Barriers................................................................................... 70

Prostate Knowledge.............................................................. 72

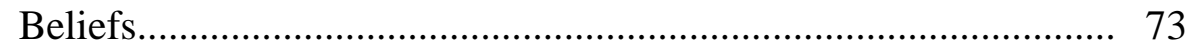

Decisional Conflict..................................................................... 76

Perceived Cost........................................................................ 76

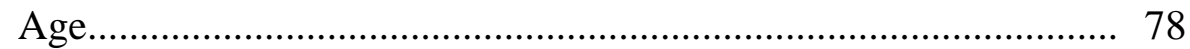

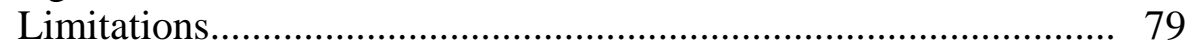

Implications...................................................................... 80

Future Research.................................................................... 83

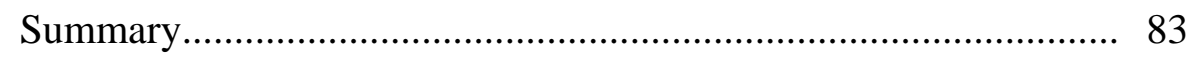

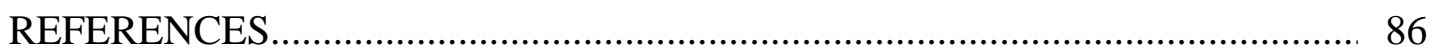

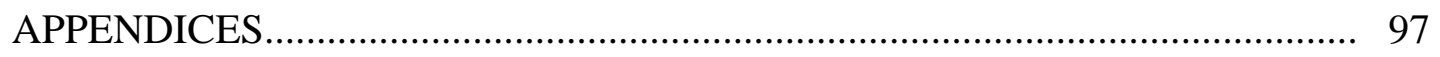

VITA 


\section{LIST OF TABLES}

TABLE

PAGE

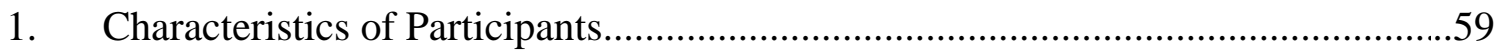

2. Personal and Family Health History Questionnaire..........................................61

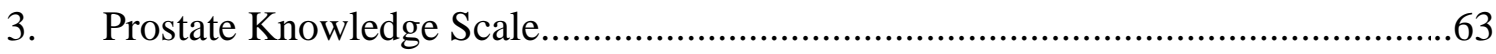

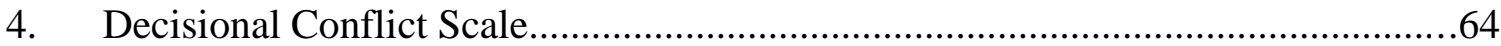

5. Barriers Ranked as One or Two for Prostate Cancer Screenings............................65

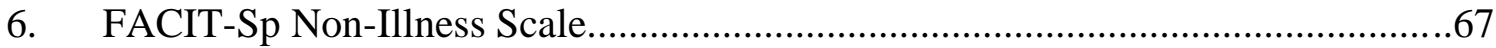

C1. Major Concepts and Empirical Indicators (see Appendix C)..............................103 


\section{CHAPTER I}

\section{INTRODUCTION}

\section{Significance of Prostate Cancer}

Approximately one in seven men in the United States will develop prostate cancer (American Cancer Society, 2015), and prostate cancer will result in death among 3\% of those diagnosed (Violette \& Saad, 2012). Prostate cancer is the number-one diagnosed non-skin cancer and one of the largest causes of mortality among men of all ethnicities in the United States (U.S. Cancer Statistics Working Group, 2014). Furlow (2012) reported the median age for diagnosis of prostate cancer in the United States is 67years old, and the median age of prostate cancer mortality is 80 years old. Approximately 220,800 new cases and 27,540 deaths related to prostate cancer are estimated for 2015 (American Cancer Society, 2015). Specifically, Wray and colleagues (2009) reported local or regional prostate cancer is diagnosed in more than $90 \%$ of cases.

The five-year survival rate approaches $100 \%$ if prostate cancer is detected in the localized stage (Wray et al., 2009). However, the five-year survival rate is only 31\% if prostate cancer is detected once it has metastasized (Jemal, Siegel, Xu, \& Ward, 2010). This contrast demonstrates the importance of detecting cancer in early stages and how the prediction of survival is closely linked to the stage of cancer at time of diagnosis.

\section{Significance of Culture}

Culture encompasses customs, beliefs, knowledge, morals, and other capabilities one possesses as part of society (Sobo, 2009). Acculturation was defined by Odedina and colleagues (2011) as the degree to which individuals from a non-dominant culture learn a host's dominant culture, including lifestyles, behaviors, languages, and values. Kumar, 
Yu, Akinremi, and Odedina (2009) explained acculturation as not only learning another culture, but also adapting to that culture. Kumar and colleagues (2009) reported behavioral factors acquired from one's culture have at times even overpowered genetic risk factors for developing prostate cancer, so cultural factors are important to study in relation to prostate cancer. This was the case for men of African descent living in various locations throughout the world, who were studied by Kumar and colleagues (2009). The researchers reported African American men had significantly higher incidence and mortality rates from prostate cancer than West African men. Additionally, African American men had a 3.5 times higher mortality rate and a 10 times elevated incidence rate than Nigerian men (Kumar et al., 2009).

Furthermore, environmental modifiable factors from one's culture or from acculturation affect prostate cancer risk (Kumar et al., 2009). Modifiable risk factors include dietary intake and physical activity (Kumar et al., 2009; Odedina et al., 2011). Kumar and colleagues (2009) compared Nigerian men $(n=121)$ who had immigrated to the United States (Texas) with indigent Nigerian men $(n=128)$ aged 35-79 using a crosssectional design, including interviews and questionnaires. The researchers found Nigerian immigrant men had significantly more hours of purposeful physical activity and dietary intake of whole grains and fruit, which could decrease prostate cancer risk. However, Nigerian immigrant men also had reported higher intake of alcohol, oils, and meats, which could increase prostate cancer risk. Kumar and colleagues (2009) concluded acculturation and adaptation to Western culture in the United States (i.e., affordability and accessibility of food and alcohol) produced differences among reported modifiable factors. 
Culture, according to Leininger (2002), is influenced by other factors besides acculturation, including communication, religion, ethnicity, and economics. Sobo (2009) found the meaning of words related to health care varied among cultures. At times, these differences led to misunderstanding and potential communication barriers with health care providers, which could in turn influence both the health actions taken, as well as the use of the health care system and its resources (Sobo, 2009).

Low knowledge levels, insufficient communication, high mortality rates, and socio-cultural factors influence prostate cancer screening practices among African American men (Wray et al., 2009). Friedman, Corwin, Dominick, and Rose (2009) used two health literacy tools to assess literacy and word choice in a sample of 25 African American men from South Carolina aged 45 and older. The researchers used interviews and discussion groups to study cultural communication and perceived barriers to prostate cancer screening. Informed decisions were observed when the material was furnished according to a man's age and language (Friedman et al., 2009).

The application of the Culture Care Diversity and Universality Theory developed by Leininger (2002) helped provide a framework for health care professionals to more accurately communicate with patients and clarify health beliefs. Also, Friedman and colleagues (2009) noted it was necessary to diminish the belief that cancer is "taboo" among African American men to discuss and to correct misperceptions concerning risks for prostate cancer. Some strategies to enact these changes included the development of culturally relevant messages and the use of multiple communication modalities, including mass media and word of mouth (Friedman et al., 2009). Furthermore, African American men participated in informed decision making concerning prostate cancer screening to a 
greater extent when educational materials were culturally appropriate and when men were guided through available options (McFall et al., 2009; Smith, Cokkinides, \& Brawley, 2012; Wolf et al., 2010).

Religion is a key component of culture (Leininger, 2002). The use of spiritually based research programs was a culturally appropriate educational strategy implemented among African American men in churches (Holt et al., 2009). Christian churches have been found to be productive settings for conducting research among African American men (Drake, Shelton, Gilligan, \& Allen, 2010; Holt et al., 2009; Jackson, Owens, Friedman, \& Hebert, 2014; Parchment, 2004) and Afro-Caribbean American men (Parchment, 2004).

Ethnicity is an aspect of culture that involves a shared origin one has with others (Sobo, 2009). The number of men diagnosed with prostate cancer varies among ethnicities (Roth, 2010), and men of African descent have an even higher mortality rate than other men (Kumar et al., 2009). Specifically, "Black” men have a 60\% higher incidence rate of prostate cancer than non-Hispanic White men and more than twice as large a mortality rate than any other ethnic group (American Cancer Society, 2015). Furthermore, men of African descent, including African American and Afro-Caribbean American men, have more aggressive prostate cancer, are 3 times more likely to be diagnosed, are diagnosed in later stages of the disease, have higher prostate-specific antigen (PSA) levels, and have earlier onset of the disease (Anderson, Marshall-Lucette, \& Webb, 2013). Men from Tobago and Jamaica have a reported incidence rate even higher than African American men (Parchment, 2004). According to the American 
Cancer Society (2015), men of African descent have the highest incidence rates of prostate cancer throughout the world.

Prostate cancer is the fifth overall cause of death and accounts for $11.1 \%$ of deaths from any type of cancer in African American men (Carter, Tippett, Anderson, \& Tameru, 2010). Carter and colleagues (2010) conducted the above-mentioned study among 76 African American men aged 45 and older in rural Alabama. Nelson, Balk, and Roth (2010) also reported African American men are diagnosed at younger ages from an archival research study in a sample of 55 African American men with a mean age of 63 . McFall and colleagues (2009) used cluster analysis and multidimensional scaling to create concept maps in a sample of 16 Hispanic and 15 African American men. The researchers found African American men have more disease symptoms and advanced stages of prostate cancer at the time of diagnosis (McFall et al., 2009).

Odedina and colleagues (2011) reported Black men born in the United States versus those born in the Caribbean or in Africa had different health behaviors and healthrelated customs concerning prostate cancer. In a sample of 2,864 men aged 40-70 labeled Black from five cities in Florida, men of African descent born in Africa discussed early detection and risk-reduction strategies concerning prostate cancer with a physician more often than men of African descent born either in the United States or in the Caribbean (Odedina et al., 2011). These findings were from self-administered surveys distributed at ethnic barbershops, meetings at faith-based organizations, or ethnic events.

Several factors contribute to the disparities noted between incidence and mortality rates for prostate cancer among African American men, including complementary and alternative medicine practices, nutrition, lack of trust in the medical system, lower 
education, health experiences from the past, poverty, poor screening habits, and family history of cancer (Jones, Steeves, \& Williams, 2010). These factors were reported by 17 African American men aged 40-71 via interviews in a qualitative study by Jones and colleagues (2010) conducted in rural Virginia. Furthermore, African American men participating in a study using focus group data collection perceived a history of unequal quality of medical treatment and decreased access to care throughout the history of the United States, thus partially explaining the disparities in mortality, incidence, and advanced disease among African American men with regard to prostate cancer (Wray et al., 2009).

Healthy People is a national program of the United States Department of Health and Human Services that sets goals in health promotion and disease prevention, focusing on various health topics every 10 years (U.S. Department of Health and Human Services, 2014). Black men in the state of Florida was the only ethnic group that did not meet the Healthy People 2010 prostate cancer goal of a mortality rate less than 28.8 per 100,000 men (Odedina et al., 2011). Since the likelihood of mortality decreases as localized early-stage prostate cancer is detected via screening participation (van Leeuwen et al., 2010), the above-noted high mortality rate disparity among Black men in Florida needs to be addressed. Odedina and colleagues (2011) found various factors influenced men's involvement in prostate cancer screening: behavioral control, knowledge, attitude, perceived susceptibility, and acculturation. Culturally appropriate strategies regarding education and interventions to promote screening should be used to help diminish this disparity regarding prostate cancer (Holt et al., 2009). 
The cost of treating prostate cancer is also significant. Patients and their loved ones have a cost of burden in regards to emotional, spiritual, physical, and social needs (Centers for Disease Control, 2011). Research is critical in order to increase prostate cancer knowledge and screening behavior, thereby reducing the cost of treatment by identifying cancer in earlier, more easily treatable stages (Aubry, Lieberthal, Willis, Bagley, \& Layton, 2013).

Prostate cancer had an overall direct cost of approximately \$12 billion in 2010 in the United States (Mariotto, Yabroff, Shao, Feuer, \& Brown, 2011). In 2020, they estimate the overall direct cost may be as much as $\$ 19$ billion. Approximately $\$ 7$ billion is spent annually on over two million prostate cancer survivors (Skolarus, Zhang, \& Hollenbeck, 2012). The cost of prostate cancer treatment alone each year is greater than $\$ 4$ billion, and by 2019, it is predicted to be $\$ 8.7$ billion (Aubry et al., 2013).

\section{Study Purpose}

The purpose of this study was to assess the likelihood of action for and factors influencing choice regarding prostate cancer screenings. It was also to discover if a relationship existed between ethnicity (African American and Afro-Caribbean American men) and knowledge of prostate health, self-efficacy (measured by men’s level of conflict in making an informed decision regarding prostate screening), perceived barriers to prostate screening, and beliefs (measured by spiritual well-being). The results were compared between African American and Afro-Caribbean American men, and differences and similarities were examined.

This study was conducted in a sample of African American and Afro-Caribbean American men. The men were from churches in South Florida that are part of the 
Congregational Health Alliance Ministry Program (CHAMP). The aforementioned CHAMP is a partnership with Baptist Health System of South Florida and several churches involved in the physical, spiritual, social, and emotional health and well-being of members in the community.

\section{Research Questions}

Three research questions were asked in order to accomplish the study purpose. The research questions were: 1 ) Is there a relationship between ethnicity (African American and Afro-Caribbean American men) and level of prostate health knowledge? 2) Is there a relationship between ethnicity (African American and Afro-Caribbean American men) and self-efficacy (measured by men's level of conflict in making an informed decision regarding prostate screening)? and 3) Is there a relationship between ethnicity (African American and Afro-Caribbean American men) and influencing factors on prostate screening behavior, including perceived barriers to screening and beliefs measured by spiritual well-being?

\section{Conceptual Framework}

The Health Belief Model (HBM) was the theoretical framework that guided this study. Rosenstock (1966) developed the model while he worked for the United States Public Health Service with the hope of studying and promoting healthy behavior and the use of health services. The original theoretical constructs of the model included perceived susceptibility, perceived severity, perceived barriers, and perceived benefits (Rosenstock, 1966). The constructs of perceptions and knowledge related to participation in health behavior and services were added to the model in the 1970s and 80s (Rosenstock, Strecher, \& Becker, 1988). Other contributing theoretical constructs and 
modifying variables were later added to the model and included perceived cost, selfefficacy, perceived control, perceived threat, cues to action, health motivation, sociopsychological variables, and demographic variables (Rosenstock et al., 1988).

Rosenstock and colleagues (1988) reported the likelihood of action, as expressed in the HBM, was affected by a modifying variable of ethnicity (i.e., culture). Additionally, that same modifying variable influenced knowledge, attitudes, and behaviors (Rosenstock et al., 1988). This study compared these same factors of knowledge, attitudes (barriers and beliefs), and behaviors (decisional conflict or selfefficacy) with a modifying variable of ethnicity between African American and AfroCaribbean American men.

A person will take a health-related action, according to the HBM, if three main components are present: the person feels a negative health condition can be avoided, the person believes if a recommended action is taken the negative health condition can be avoided, and the person believes he/she can take action with favorable outcomes (Janz \& Becker, 1984). Another main objective of the HBM is to explain beliefs and attitudes that impact adherence to suggested medical care and participation in preventative health measures (Dossey \& Keegan, 2009). The HBM expresses how expectations (belief and knowledge), perception of threat, and cues to action (internal and external motivators) affect one another (Rosenstock et al., 1988).

In an intervention study, participants who had higher perception of risk and knowledge had a significant increase in prostate cancer screening behavior (Emerson, Reece, Levine, Hull, \& Husaini, 2009). Emerson and colleagues (2009) conducted the study among African American men $(n=152)$ aged 40-70 in 45 churches in Tennessee 
as a quasi-experimental, delayed-control (cross-over) design study with randomization at the church level. The investigators used the HBM as a framework for the study. Interviews were conducted by an African American man and self-reported questionnaires were distributed regarding prostate cancer knowledge, attitudes, beliefs, and screening behaviors. Data were collected at baseline and then at three-month and six-month follow-ups after an hour-long educational intervention consisting of a 10-minute video and a physician’s lecture and counseling (Emerson et al., 2009).

Furthermore, high perceived risk for cancer was related to an increase in healthseeking behavior for colon, breast, and prostate cancer screenings in a sample of 1,729 English-speaking men and women of various ethnicities aged 40 years and older from the United States (Dillard, Couper, \& Zikmund-Fisher, 2010). Dillard and colleagues (2010) used random-digit dialed telephone surveys and the HBM as a framework for the study. The researchers found among men with high-perceived risk, 35\% sought screening versus only $18 \%$ among those who had low-perceived risk. Additionally, self-administered surveys were distributed by Odedina and colleagues (2011) among Black men, including African American and Afro-Caribbean American men in Florida, aged 40-70, using the HBM as a framework. Odedina and colleagues (2011) found perceived behavioral control, knowledge, acculturation, perceived susceptibility, and attitude influenced prostate cancer screening participation.

\section{Study Variables for the Framework}

The HBM was used in this study to help explain the preventative health behavior of prostate cancer screening. Three theoretical constructs from the HBM, including knowledge, self-efficacy (measured by decisional conflict), and perceived barriers, were 
examined in this study. These constructs were examined as dependent variables to the independent variable of ethnicity, which is a fourth theoretical construct from the HBM under the heading of modifying variables. Spiritual well-being, which is not a theoretical construct of the HBM, was another dependent variable examined in connection with ethnicity. 


\section{CHAPTER II}

\section{REVIEW OF THE LITERATURE}

The literature review was conducted via a search of electronic databases that included Cumulative Index to Nursing and Allied Health Literature (CINAHL) and PubMed. The search was limited to research reports in English from 2009 to 2015. The key words used to identify related literature from CINAHL were ("Prostatic Neoplasms") AND (MH "Cancer Screening") AND (consequence* OR importan* OR barrier* OR obstacle* OR impact* OR value* OR cultur* OR belie*). "Prostatic Neoplasms"[Mesh] OR “prostate cancer” OR “prostatic neoplasms” AND "Early Detection of Cancer"[Mesh] OR Screen* OR “early detection of cancer” OR “Cancer Early Diagnosis” OR “Cancer Early Detection” OR “Early Diagnosis of Cancer” AND "West Indies"[Mesh] OR "African Continental Ancestry Group"[Mesh] OR "African Americans"[Mesh] OR “Afro Caribbean” OR black* OR “African American” OR Jamaica* OR Haiti* OR Trinidad* OR Tobago* OR "Caribbean black” OR "Caribbean American” OR “West Indies” AND "Data Collection"[Mesh] OR "Questionnaires"[Mesh] OR survey* OR questionnaire* OR “data collection” AND "Patient Acceptance of Health Care"[Mesh] OR "Attitude to Health"[Mesh] OR "Health Services Accessibility"[Mesh] OR "Health Knowledge, Attitudes, Practice"[Mesh] OR consequence* OR importan* OR barrier* OR obstacle* OR impact* OR value* OR belie* OR knowledge OR cost OR attitude* OR spiritual* OR “comfort level” OR “Informed Decision Making” OR access* OR aware* were used for PubMed. 


\section{Prevalence and Risk for Men of African Descent}

The age-adjusted (a strategy to proportionally compare populations with different age distributions) prostate cancer count during 2009-2011 in Florida was 40,824 and the rate was 115.8 per 100,000 men, but Miami-Dade County had an even higher overall rate of 127.8 per 100,000 men and a count of 4,929 (University of Miami (FL) Medical School, Florida Cancer Data System, 2008-2011). The age-adjusted prostate cancer count for Black men, including African American and Afro-Caribbean American men, during 2008-2010 for Miami-Dade County was 1,086 and a rate of 195.8 per 100,000 men, as compared to "White" men with a rate of 119.3 per 100,000 men (University of Miami (FL) Medical School, Florida Cancer Data System, 2008-2011). It was found to be important to include Afro-Caribbean American men in studies among Black men in South Florida because a large number of Afro-Caribbean American men have immigrated to that part of the United States due to its similar climate and close proximity to the Caribbean (Archibald, 2011).

The ethnic group labeled Black American men was reported as having low prostate cancer survival rates, more late-stage prostate cancer diagnoses, incorrect identification of early symptoms of prostate cancer, and a prostate cancer mortality rate greater than 28.8 per 100,000 men in the state of Florida (Odedina et al., 2011). Odedina and colleagues (2011) reported several reasons for prostate cancer disparities among Black American men, including inadequate knowledge, lack of trust of the health care system, lower socioeconomic status, and limited health care access. These researchers collected data from 2,864 men aged 40-70 labeled Black, including African American and Afro-Caribbean American men, in five cities in Florida. 
African American men specifically had higher mortality and incidence rates than any other ethnic group (Lehto, Song, Stein, \& Coleman-Burns 2010; Nelson et al., 2010). Yet when compared with other ethnic groups, African American men also had a lower perceived risk of being diagnosed with prostate cancer (Glenn et al., 2012). Glenn and colleagues (2012) reported this finding from the study, which utilized telephone surveys among first-degree relatives $(n=1,029)$ of men diagnosed with prostate cancer (354 nonLatino White, 228 Latino, 272 African American, and 175 Asian men). Additionally, African American men exhibited low rates of participation in cancer research (Friedman et al., 2012). The American Cancer Society (2015) suggested African American men could be at a higher risk for prostate cancer diagnosis due to genetics, inherited conditions, diet, and family history of prostate cancer.

Odedina and colleagues (2011) studied differences among men of African descent born in the United States versus men born in Africa and born in the Caribbean. Differences existed in early detection and risk-reduction behaviors related to prostate cancer. Odedina and colleagues (2011) found Black men born in the United States had the highest knowledge level regarding prostate health and were the most likely to have health insurance, as compared to African-born and Caribbean-born Black men. Black men born in Africa discussed prostate cancer risk reduction and early detection with a health care provider the most (Odedina et al., 2011). In a study of 180 Black men, Jamaican-born American men had higher prostate cancer anxiety than African American men born in the United States (Consedine, 2012). In addition, men from Tobago and Jamaica had a higher incidence rate than African American men (Parchment, 2004). 
However, Afro-Caribbean American men had similar findings as African American men in several areas: both had more aggressive prostate cancer, were 3 times more likely to be diagnosed, were diagnosed in later stages of the disease, had earlier onset of the disease, had higher PSA levels, and had a 60\% higher incidence rate than any other ethnic group (Anderson et al., 2013). Men of African descent from Caribbean countries, similar to African American men, also had over twice as high mortality rates from prostate cancer (Ng et al., 2013). Furthermore, African American and AfroCaribbean American men (specifically subpopulations of immigrant Caribbean men) had low rates of participation in PSA screenings (Lee, Consedine, Gonzalez, \& Spencer, 2012). Ng and colleagues (2013) indicated more studies should be conducted among Afro-Caribbean American men because not as many studies have been conducted as among African American men.

\section{Screening}

\section{Screening Properties}

The major objective in screening for prostate cancer is to identify cancer in as early a stage as possible because treatment in an early stage has been shown to decrease both mortality and metastasis (van Leeuwen et al., 2010). Screening of prostate cancer is most commonly done by a digital rectal exam (DRE) and a PSA test (Wong \& Coups, 2011). A DRE is performed by a health care provider inserting a gloved, lubricated finger into the rectum to palpate for abnormalities of the prostate (Wray et al., 2009). The DREs have a wide specificity and sensitivity range, which could be explained in part by the anatomical position and volume of the prostate gland (Koulikov, Mamber, Fridmans, Arafeh, \& Shenfeld, 2012). Also, Koulikov and colleagues (2012) concluded 
a DRE exam had a high inter-observer variability due to the subjective opinion and length of finger of the health care provider. Furthermore, Ugwumba and Nnabugwu (2012) reported the examiner needed to have performed several DREs in order to be proficient in conducting a DRE, and supervision and models could enhance the ability of a new examiner to conduct an accurate DRE.

The prostate gland's epithelial cells produce an elevated level of the glycoprotein PSA in the presence of prostate tissue damage. Richard Ablin invented the PSA test and recommended that due to its low sensitivity and specificity for prostate cancer, the PSA test should be used in conjunction with another screening modality, such as a DRE (Bul \& Schrööder, 2011). Testing for PSA levels was first approved for use in combination with a DRE in men 50 years old or older to screen for prostate cancer by the United States Food and Drug Administration in 1994 (Austin, 2012). Bul and Schrööder (2011) conducted a non-systematic review of evidence both for and against prostate cancer screening with a particular emphasis on three randomized screening trials. The researchers concluded the ideal screening tool for prostate cancer did not exist. Such a tool could be used by doctors to help doctors avoid misdiagnosing indolent cancers that would never cause problems or symptoms, as well as detect harmful cancers in an early and more easily treatable stage (Aubry et al., 2013; Bul \& Schrööder, 2011). To improve prostate cancer screening in the future, PSA tests may include PSA molecular biomarkers or other forms of testing as well (Shariat et al., 2011).

According to Soydan and colleagues (2013), one way to avoid treating indolent cancers was to use active surveillance, especially for men with low-risk prostate cancer. During active surveillance, practitioners wait to observe any symptoms that arise or any 
drastic changes that occur between annual screenings in order to avoid unnecessary treatment of benign prostatic conditions, instead of taking further action in response to a high PSA level (Soydan et al., 2013). Active surveillance not only evades unnecessary treatments, but active surveillance also detects higher risk prostate cancers that need to be treated (Soydan et al., 2013). It is important for both practitioners and patients to understand active surveillance incorporates regular examination of patients with low-risk prostate cancer. Soydan and colleagues (2013) reported the screening examinations involved in active surveillance are PSA-level measurements, DREs, and sometimes image guided biopsies. Cooperberg, Carroll, and Klotz (2011) discovered two challenges to the acceptance of active surveillance use by health care professionals and patients: understanding what the end goals were for the surveillance and the risk of progression at the time of diagnosis. When active surveillance was used among men diagnosed with low-risk prostate cancer, cure rates did not change for men treated later as compared to men who were treated initially after diagnosis (Cooperberg et al., 2011).

\section{Why Screening is Important}

Van Leeuwen and colleagues (2010) reported when PSA screenings were conducted, prostate cancer metastasis was reduced by $53 \%$ and prostate cancer mortality was decreased by $37 \%$ over the eight-and-one-half-year period of the study. In fact, Wray and colleagues (2009) reported screening as the only strategy with a reasonable reduction in prostate mortality. The ability to detect prostate cancer early and thus decrease mortality risk demonstrates the importance of implementing prostate screenings (Basch et al., 2012). 
A total of $51.1 \%$ of men 50 years old or older in the United States in 2010 reported participating in a PSA test and 55.4\% had a DRE (Wong \& Coups, 2011). Lack of knowledge regarding prostate cancer and low rates of screening have been observed across the United States among various ethnicities (Rivera-Ramos \& Buki, 2011). Screenings are participated in less when men have a low perceived risk of developing and dying from prostate cancer (Shavers, Underwood, \& Moser, 2009). The researchers selected a sample of 1,075 using random-digit-dialing, which consisted of African American, Hispanic, and Caucasian males aged 45 or older without any history of prostate cancer. An analysis was conducted examining the association among perception of risk of developing prostate cancer, demographic characteristics, and PSA test utilization among the sample population using a multivariable logistic regression analysis.

Shavers and colleagues (2009) found reasons for low screening rates included low perceived personal risk for cancer diagnosis, the role race/ethnicity played on these perceptions, how information was processed regarding statistical cancer risks, and processing methods. The findings contributed to the body of knowledge on men's perception concerning prostate cancer risk and demonstrated that providing information regarding risk of developing prostate cancer could aid all men, especially African American men, in making a decision regarding prostate cancer screening (Shavers et al., 2009). Furthermore, McFall and colleagues (2009), through the use of concept mapping, found a high importance rating was necessary for informed decision making concerning prostate cancer screening in African American men. 


\section{Screening Controversy}

Prostate cancer screening using the PSA test has decreased the age of diagnosis (Cross, Ritter, \& Reding, 2012) and mortality rate and increased the early detection and treatment of prostate cancer (van Leeuwen et al., 2010). However, a controversy exists regarding PSA testing due to the over diagnosis and over treatment of indolent prostate cancer (Bul \& Schrööder, 2011). The controversy has been debated in the United States and other countries for decades (Austin, 2012). The controversy mainly involves investigating the bioethical issue of the benefits of screening versus the harms and subsequent possible treatment and side effects (Davis et al., 2012).

The United States Preventive Services Task Force (USPSTF) does not recommend mass prostate cancer screenings of the general male population. The USPSTF's report from 2011 concluded PSA screenings either did not reduce prostate cancer mortality or only reduced mortality to a small degree, and PSA screenings resulted in needless subsequent evaluation and treatments (Chou et al., 2011). The USPSTF also concluded risks outweighed benefits with at least moderate confidence for men 75 years or older. However, insufficient evidence was available to either recommend for or against routine screening in men less than 75 years old (Howard, Tangka, Guy, Ekwueme, \& Lipscomb, 2013). Howard and colleagues (2013) noted 7.9\% fewer PSA tests were completed among men 75 years old or older over the two years following the revised recommendation.

Because other non-cancer-related factors may contribute to high PSA levels, insufficient evidence was available to recommend for or against PSA screening. Noncancer related factors include infection, inflammation, benign prostatic hyperplasia, and 
other benign states (Howard et al., 2013). Aubry and colleagues (2013) reported out of the 1.3 million biopsies performed annually in the United States, $75 \%$ were negative. Many men subsequently were subjected to an unnecessary invasive biopsy procedure with its own inherent risks (Aubry et al., 2013).

In addition, Bell and Kazanjian (2011) reported prostate cancer progresses very slowly, so most men did not experience serious symptoms or a premature death from the disease. Davis and colleagues (2012) concluded some screenings have detected insignificant prostate nodules and indolent prostate cancer. It was also reported surgeries have been performed at times unnecessarily. Sometimes, side effects are needlessly experienced in association with the treatment of prostate cancer: physiological dysfunctions (i.e., urinary and bowel), sexual dysfunctions, and emotional dysfunctions (Davis et al., 2012). A meta-analysis by Djulbegovic and colleagues (2010) and a randomized screening trial by Sandblom, Varenhorst, Rosell, Lofman, and Carlsson (2011) both failed to report a decrease in mortality with the implementation of PSA screenings; therefore, both groups of researchers could not support or refute routine prostate cancer screening.

Detractors of PSA testing point to over diagnosing and unnecessary procedures (Ilic, Neuberger, Djulbegovic, \& Dahm, 2013). Bul and Schrööder (2011) concluded PSA measurements were not accurate to screen for prostate cancer; therefore, PSA measurements were not to be used alone in the screening process. The researchers also discovered even if the PSA level was low, sometimes a high-grade prostate cancer was still present. Bul and Schrööder (2011) found approximately 50\% of the men who were identified as having prostate cancer, through prostate cancer screening, never experienced 
prostate cancer symptoms throughout life. Harms of participating in screening were reported as over diagnosis and overtreatment of false positive PSA results and negative effects from biopsies, including pain, bleeding, and infection (Ilic et al., 2013).

However, supporters of PSA testing pointed to other research that showed screening for prostate cancer had the potential to prevent mortality and metastasis. For example, participating in PSA screening reduced prostate cancer metastasis by $53 \%$ and prostate cancer mortality by $37 \%$ during a study period of eight and one-half years (van Leeuwen et al., 2010). Likewise, Bul and Schrööder (2011) found screening decreased mortality at a nine-year follow-up by $20-31 \%$ and $44-56 \%$ at a 14 -year follow-up in the Göteborg screening trial in the European Randomized Study of Screening for Prostate Cancer, which was a trial involving seven countries and over 180,000 men.

For men at risk for prostate cancer, the American Urological Association (AUA) recommends routine screening with a PSA test and a DRE (Aubry et al., 2013). The AUA firmly supports this recommendation because screenings have assisted in the diagnosis of early low-stage cancers. Without PSA and DRE routine screenings, the AUA reports prostate cancer will have increased cost for treatment, side effects, burden from the disease, and aggressive treatments for high-risk, late-stage cancers (Aubry et al., 2013). Early detection from screenings decreased treatment costs and increased curative rates in a study by Skolarus and colleagues (2012). Increased early low-stage cancer diagnosis of the disease was a main benefit from prostate cancer screenings (Eldefrawy, Katkoori, Abramowitz, Soloway, \& Manoharan, 2013). Since the implementation of prostate cancer screenings, mortality rates from prostate cancer have decreased (McCormick, Osman, \& Pomerantz, 2010). 
Evidence exists for both detractors and supporters of routine prostate cancer screening among the general male population in the United States, as well as specific recommendations for certain groups of men. For example, Basch and colleagues (2012) suggested men with less than a 10-year life expectancy not be encouraged to participate in a PSA test due to the risk of a false positive, subsequent biopsy, and unnecessary treatment. The American Cancer Society (2015) also recommended men who are not expected to live at least 10 years not be screened because prostate cancer spreads so gradually. However, men who have a greater than 10-year life expectancy were encouraged to discuss with health care providers the benefits and harms of PSA testing, starting at age 50, if they are at average risk for prostate cancer (American Cancer Society, 2015).

Other specific screening recommendations were made for men with relatives with prostate cancer by McDowell, Occhipinti, Gardiner, Baade, and Steginga (2009). The researchers found family history of cancer does not only increase risk of cancer in men of African descent, but in all men. The risk of a first-degree relative of a man with prostate cancer being diagnosed with prostate cancer was more than twice that of the general population, and the risk was 3 times greater if more than one first-degree relative was diagnosed with prostate cancer. An increased risk was also noted if the relative was less than age 60 when he was diagnosed (McDowell et al., 2009).

In light of these increased risks, the American Cancer Society (2015) recommended discussing risks and benefits concerning screening at age 45 for African American males or for men with a first-degree relative with an early age (less than 65 years old) diagnosis of prostate cancer, and at age 40 for men with multiple first-degree 
relatives diagnosed with prostate cancer at an early age. After this discussion, if a man decided to participate in screening, the American Cancer Society (2015) recommended a PSA test be completed either alone or with a DRE. If a man was unable to decide, it was suggested the health care provider make the decision of screening or not based on the patient's values and health preferences. If, after testing, the PSA was less than $2.5 \mathrm{ng} / \mathrm{ml}$, rescreening was not necessary for two years; and if greater than $2.5 \mathrm{ng} / \mathrm{ml}$, rescreening was recommended annually (American Cancer Society, 2015). Because men of African descent had higher rates of both diagnosis of and mortality from prostate cancer, it was recommended screening be done early and frequently for possible diagnosis and treatment (American Cancer Society, 2015).

The consensus on the screening controversy was that a PSA test should not be used alone for screening, but should be used in conjunction with a DRE for more accurate results (Smith et al., 2012; Vickers, Roobol, \& Lilja, 2012). Also, it was agreed upon that prostate cancer screenings should incorporate men participating in an informed decision-making process with health care providers (American Cancer Society, 2015). Informed decision making involved two main parts: 1) obtaining information regarding risks, benefits, alternatives, and uncertainties regarding prostate cancer from a health care provider and culturally reliable and appropriate resources and 2) making the decision according to the man's values and preferences (Davis et al., 2012). Ferrante, Shaw, and Scott (2011) found shared decision making was a method used by providers to instruct men concerning benefits, possible harms, limitations, and risks of prostate cancer and screenings, and then to help men make a choice based on these issues and a man's beliefs, values, and desires. Additionally, Ferrant and colleagues (2011) found informing 
men of the benefits and limitations of prostate cancer screening was a more effective means to promote and facilitate shared decision making in regards to screening.

\section{Perceived Barriers to Screenings}

Even though screenings have decreased prostate cancer metastasis and mortality (van Leeuwen et al., 2010), several barriers have been found to decrease men’s participation in prostate cancer screenings. The impediments to health-seeking behaviors with respect to prostate cancer screenings were significant. Different barriers were noted among racial groups.

Some barriers specific to African American men included low knowledge, understanding, and information regarding prostate cancer screenings, such as a misunderstanding between diagnostic tests and screening (Friedman et al., 2009). Lack of trust in the health care system, deficient communication with health care providers, inconvenient clinic hours, limited access to health care services, and lack of health insurance were also common barriers reported by African American men (Friedman et al., 2009). Friedman and colleagues (2009) used two health literacy tools in a sample of 25 African American men from South Carolina aged 45 and older and found the men had adequate health literacy levels. The authors concluded health literacy levels among African American men were not found to influence screenings. However, the men were not able to articulate prostate cancer risk factors and preventive behaviors during interviews and discussion groups. Friedman and colleagues (2009) suggested that, in the future, prostate cancer information be presented without extensive medical jargon but with culturally appropriate messages to help African American men understand the information more completely, regardless of what the health literacy scores showed. 
Emerson and colleagues (2009) conducted a study utilizing interviews and selfreported questionnaires in churches among 152 African American men aged 40-70 and found a significant barrier to screening was deficient knowledge of where to access affordable treatment if cancer was found. Likewise, Oliver, Grindel, DeCoster, Ford, and Martin (2011) reported in a sample consisting of predominantly African American men, other barriers were: the screening taking too much time at $87.1 \%$, embarrassment at $74.2 \%$, lack of understanding of the screening process at $68.7 \%$, and fear of pain related to the screening at $66 \%$.

Wray and colleagues (2009) reported African American men had the barrier of decreased trust in the health care system, which stems from unequal quality of care and limited access to care among African Americans. The researchers conducted five focus groups (four with healthy men and one with prostate cancer survivors) among African American men, led semi-structured interviews with 19 community leaders, and evaluated two outreach projects involving survivors in which they discussed prostate cancer and screening with African American men. The researchers reported barriers to prostate cancer screening and treatment among African American men in three main levels (Wray et al., 2009). The first was at the individual level, with fear of prostate cancer and deficient knowledge of prevention, the condition, and treatment. The second was at the socio-cultural level, which consisted of such obstacles as unwillingness to discuss cancer, lack of a provider to perform preventive and routine care, dislike of screening, and lack of trust of the medical system. The third was at the institutional level, with insufficient educational programs focused on prostate cancer. 
Barriers specific to Afro-Caribbean American men were gender-related perceptions, concerns for privacy, time away from work, limited access to care, and ineffective dissemination of health information (Ng et al., 2013). These barriers to health-seeking behaviors, including participation in prostate cancer screenings, were discovered after completing key informant interviews among 30 African Barbadian men, wives of Barbadian men, health care providers, and community and religious leaders. Additionally, fear was a screening barrier Kleier (2010) reported via surveys in a sample of 143 Afro-Caribbean (Haitian) American men.

In-depth, face-to-face interviews were conducted by Anderson and colleagues (2013) with seven Afro-Caribbean men in the United Kingdom, and four major themes in regards to prostate cancer awareness, barriers, and screening beliefs were identified: disease-prompted awareness, checking up as a necessary evil, defining and constructing factors influencing prostate cancer screening, and appraising perceived myths of prostate cancer. These four major themes helped explain Afro-Caribbean men's perspectives of prostate cancer awareness, barriers to screening, factors influencing screening beliefs, and fears of the disease. The authors noted professional background and education influenced the level of awareness of prostate cancer and willingness to seek help. The authors also found specific health education strategies targeting the Afro-Caribbean population could encourage men to seek prostate health assistance earlier.

Studies were also conducted with both African American and Afro-Caribbean American men in regards to prostate cancer screening barriers. In a study involving 2,864 men aged 40-70 of African descent, Odedina and colleagues (2009) found inadequate knowledge of prostate cancer disease and screening, lower socioeconomic 
status, and incorrectly identified early symptoms of prostate cancer were barriers to screening. Additionally, prostate cancer screening participation was influenced by perceived behavioral control, acculturation, perceived susceptibility, and attitude (Odedina et al., 2011).

Lee and colleagues (2012) indicated African American and Afro-Caribbean American men from Jamaica and Trinidad participated less in DRE screenings, even after adjusting for comorbidities, access to care, and socioeconomic status, and had significantly higher levels of health care system barriers. These barriers included greater prostate cancer worry, fear of screening, and difficulty navigating the health care system. Barriers to screening from men of African descent, including Afro-Caribbean American men from Jamaica, Bahamas, Trinidad, Haiti, and Guyana, were embarrassment to have a DRE and fear of impotence and incontinence if diagnosed with and treated for prostate cancer (Parchment, 2004).

Pedersen, Armes, and Ream (2012) conducted a systematic review of 13 qualitative studies and 20 cross-sectional surveys to discover the factors influencing prostate cancer participation among African American and Afro-Caribbean American men. Awareness of the personal risk for prostate cancer was not consistent among the studies. Mistrust of the health care system, limited access to health care, lack of trusting relationships with health professionals, misunderstanding regarding methods of diagnosis and treatment, and feeling a threat to a man's sense of masculinity were common barriers. Researchers should be aware of reported perceived barriers when communicating prostate health and screening information with African American and Afro-Caribbean American men to decrease these barriers (Pedersen et al., 2012). 
Methods have been studied to reduce prostate cancer screening barriers among men of various ethnicities. Increasing perceived risk of prostate cancer, participating in making decisions, and educating men regarding prostate cancer were methods to overcome barriers (Dillard et al., 2010). Dillard and colleagues (2010) found these methods by conducting telephone surveys among 1,729 English-speaking men and women aged 40 years old and older of various ethnicities from the United States.

Other methods to reduce prostate cancer screening barriers among African American men have been discovered as well. Wray and colleagues (2009) collected evaluations from African American men and found a successful way to increase prostate cancer knowledge, promote conversations centered on prostate cancer, and encourage men to engage in screenings was having survivors lead outreach projects among African American men. Similarly, Mitchell (2011) found from a survey distributed at a community health fair among 229 African American men 18 years old or older higher educational levels, access to a health care provider on a regular basis, and older age were all associated with increased rates of prostate cancer screening. Additionally, greater access to care, education, insurance coverage, knowledge, and community involvement increased the number of African American men who completed health screenings and increased the perception of risk for prostate cancer (Emerson et al., 2009).

Furthermore, prostate cancer screening barriers among African American men were reduced when perceived risk of prostate cancer diagnosis was increased. Lehto and colleagues (2010) reported a correlation between perceived risk of prostate cancer and increased participation in screenings. The findings were from a study of African American men $(n=60)$ aged 38 to 79 recruited from churches in the greater Detroit 
metropolitan area. The self-administered survey results revealed health values and age were the two most predictive variables impacting prostate cancer screening among African American males (Lehto et al., 2010). Also, Lehto and colleagues (2010) concluded educational interventions focused on health values and screening interventions should be aimed at younger African American males. Shavers and colleagues (2009) reported men who had a lower perceived risk of the disease were less willing to participate in prostate cancer screenings, and African American men often perceived prostate cancer diagnosis risk less than the average man the same age. Shavers and colleagues (2009) conducted the study among African American, Hispanic, and nonHispanic White $(n=1,075)$ men aged 45 or older, who responded to the 2003 Health Information National Trends Study (HINTS).

\section{Culture}

\section{Importance of Culture in Research}

Cultural issues are complex and need to be addressed in research to improve the health care system (Sobo, 2009). Economic, political, and social factors were noted to be interconnected with cultural issues. For example, the economical concern of cost has been observed to be related to cultural understanding of the health care system. Cost of treatment for prostate cancer was reduced as knowledge of prostate health and screening behavior increased (Aubry et al., 2013). Also, the Patient Protection and Affordable Care Act is a political aspect of culture that has expanded health insurance coverage to millions of Americans and made it possible for increased access to and understanding of the health care system (Andrews, Darnell, Mcbride, \& Gehlert, 2013). Additionally, social support, a social component of culture, was reported by Jones and colleagues 
(2010) to increase informed decision making regarding prostate screenings in the health care system. Furthermore, Sobo (2009) reported meanings of words or semantics varied among cultures regarding health care, illness, and well-being. These differences could cause miscommunication and misunderstanding, which could affect how resources were used and actions were made. Additionally, culturally appropriate communication was essential for culturally competent care (Sobo, 2009).

The concept of cultural competence includes providing care that is not only culturally responsive, effective, and relevant, but also improves health outcomes and decreases ethnic and racial disparities (Shen, 2015). Cultural competence is one cultural issue that has been extensively studied in nursing over the past 30 years, and various models and instruments have been developed to measure cultural competence (Shen, 2015). Cross, Bazron, Dennis, and Isaacs (1989) first used the term cultural competence in published literature as, "a set of congruent behaviors, attitudes, and policies that come together in a system, agency, or amongst professionals and enable that system, agency, or those professionals to work effectively in cross-cultural situations” (p. 7).

Culturally congruent care (often referred to as cultural competence) for nursing was defined by Leininger (2002) to include religion, economics, language, history, worldview, gender, practices, meanings, values, environment, symbols, politics, and expressions. Leininger (2002) was the first to study culturally congruent care connecting nursing with anthropology and developed the Culture Care Diversity and Universality Theory, the use of transcultural nursing practice, and the use of ethnonursing research. The theory was culturally specific, but also was broad and applicable to various cultures on health, death, illness, and well-being for groups and individuals (Leininger, 2002). 
Leininger's (2002) goal for the theory was to develop safe, meaningful, and culturally congruent care for both diverse and similar cultures based on research. The application of the theory caused improvements to the health care system, such as more accurate communication between patients and health care professionals (Leininger, 2002). Shen (2015) reported that the cultural, racial, and ethnic diversity in the United States has sparked a need for a variety of culturally competent models in nursing to be developed and promoted. Cultural competency has increased understanding, awareness, and sensitivity of various cultures among nurses and other health care providers during interactions with patients and has increased the ability to provide quality competent care (Shen, 2015).

\section{Culturally Appropriate Strategies}

Miller (2014) stressed additional culturally tailored outreach efforts needed to be made and further research needed to be conducted in order to understand knowledge, attitudes, and behaviors in terms of prostate cancer screening among African American men. Those conclusions were drawn after a study was completed among 46 African American men aged 30-45 from urban Ohio. Culturally appropriate strategies to help diminish disparities in men's health were of even greater importance with African American men than with other ethnic groups (Holt et al., 2009). A successful educational method concerning prostate health was using culturally appropriate strategies with African American men from a study conducted at two Baptist churches in Alabama among 49 African American men aged 45 or older (Holt et al., 2009). Two main components needed to increase the number of African American men completing health screenings were education and community involvement (Emerson et al., 2009). 
Making the educational materials culturally appropriate and the manner in which those materials were presented were also important strategies. Drake and colleagues (2010) noted a significant improvement $(\mathrm{p}=0.025)$ in African American men's ability to make informed decisions regarding early detection for prostate cancer when accurate and meaningful information was received from an educational church-based intervention among predominantly African American men $(n=73)$. Making educational material culturally appropriate helped African American men comprehend screening options and make informed decisions regarding prostate cancer (McFall et al., 2009; Smith et al., 2012; Wolf et al., 2010). An increased number of informed decisions regarding prostate cancer screening were demonstrated by African American men when material was accommodated to a man's language and age (Friedman et al., 2009). Archibald (2011) found culturally appropriate strategies specifically for Afro-Caribbean American men through a content analysis. Among Afro-Caribbean American men, strategies should focus on the men's healthy intentions, appropriate attitudes, and strong values. These three elements were reported as necessary components to consider when developing an intervention for this population (Archibald, 2011).

Another culturally appropriate strategy was changing the setting of prostate education from a clinical one to a community one (Drake et al., 2010). In fact, Drake and colleagues (2010) completed an educational church-based intervention among 73 men who were predominantly African American and reported churches were successful community settings for educating African American men regarding prostate cancer knowledge and screening. Using community involvement, especially using churches as settings, has opened new doors for African American men to be educated and receive 
health care in settings other than the traditional, clinical ones (Jackson et al., 2014).

Saunders and colleagues (2013) concluded the majority of African American men were highly involved in churches, so churches appeared to be good settings for conducting research in this population. Christian churches have proven successful locations for surveying and educating African American men (Holt et al., 2009; Jackson et al., 2014; Parchment, 2004) and Afro-Caribbean American men (Parchment, 2004) regarding health issues and practices in other studies as well.

\section{Prostate Cancer Cost}

The overall cancer cost in 2010 in the United States was estimated by the Centers for Disease Control (2011) as a combination of lost productivity and medical costs at \$263.8 billion. Furthermore, Mariotto and colleagues (2011) reported prostate cancer had a direct cost of $\$ 12$ billion in 2010 in the United States. They estimated the direct cost may increase to $\$ 19$ billion in 2020. Negative effects on quality of life were reported as indirect costs related to prostate cancer (Aubry et al., 2013). The annual cost of prostate cancer treatment in 2019 was predicted to be $\$ 8.7$ billion (Aubry et al., 2013). Eldefrawy and colleagues (2013) reported prostate cancer cost increased in recent years in the United States due to earlier detection, higher incidence, and longer life expectancy.

Aubry and colleagues (2013) listed screening cost as one component of prostate cancer cost. The risk of a false positive result from a PSA test was an additional cost (McCormick et al., 2010). Annually, 19 million men were screened for prostate cancer using PSA levels in the United States with an estimated cost of $\$ 1.86$ billion (Aubry et al., 2013). Also, from these 19 million men screened, it was observed 4.7 million had abnormal PSA results ( $\geq 4.0 \mathrm{ng} / \mathrm{mL})$. 
The Patient Protection and Affordable Care Act does not guarantee all people qualify for the same preventive services, including prostate cancer screenings (Wilensky \& Gray, 2013). However, Congress has developed some requirements for Medicare, Medicaid, and private insurers under the Patient Protection and Affordable Care Act to waive the coinsurance and deductible payment through the Social Security Act for PSA screenings but not for DRE screenings (Stranne, 2011). Another aim of the Patient Protection and Affordable Care Act was to make insurance more affordable and in turn decrease the number of uninsured in America (Giaimo, 2013). The Act expanded coverage to approximately 30 million Americans (Andrews et al., 2013). Goldenberg (2013) predicted that by 2019, a total of 17 million Americans below the poverty line would be eligible for Medicaid and receive improved access to wellness visits and free preventative care, including prostate cancer screenings on all insurance plans. Just as people do not always take advantage of Medicaid when it is available, men frequently did not take advantage of prostate screening even when screenings were free (Gash \& McIntosh, 2013).

Aubry and colleagues (2013) noted another cost was from the conducting of biopsies, which were invasive and possibly harmful. Aubry and colleagues (2013) reported 1.3 million biopsies (one biopsy was defined as one biopsy session, which included 10 to 12 needle core tissue samples) were performed annually. The researchers found $75 \%$ of men had negative biopsies, and multiple biopsies increased a patient's risk of infection, hypotension, sepsis, discomfort, cystitis, endocarditis, prostatitis, urinary symptoms (including hematuria), antibiotic resistance, and gastrointestinal hemorrhage. 
Prostate cancer treatment choice also affects cost. Eldefrawy and colleagues (2013) conducted a comparison of different treatment options for prostate cancer of the cumulative medical costs for open radical retropubic prostatectomy, robotic-assisted radical prostatectomy, external beam radiotherapy, brachytherapy, and active surveillance. Active surveillance had a low initial cost, was the overall most costeffective choice, and had the lowest follow-up cost in treatment of low-grade prostate cancer. Active surveillance was found to reduce treatment interventions for prostate cancer; therefore, it was found to decrease treatment complications and the effects on quality of life. Also, when outpatient centers were used for active surveillance, the costs from lost days from work, including loss of wages and decreased productivity and use of inpatient facilities, were reported to be minimal (Eldefrawy et al., 2013).

Bul and Schrööder (2011) suggested several factors needed to be combined to assess the value of prostate cancer screening. Additionally, the authors reported benefits, costs, and QALYs were components that should all be compared to understand the possible reduction in prostate cancer mortality by men participating in screenings. In addition, various treatment options exist that differ in price, subsequent QALYs, and costs and benefits for the patient (Nguyen et al., 2011). Not only was the patient affected, but his friends, caregivers, and family members also had a cost of emotional, spiritual, physical, and social burden placed on them (Centers for Disease Control, 2011). Indeed, cancer cost is calculated not only in a dollar amount, but in burden and effects on quality of life.

Some symptoms that affected quality of life included erectile dysfunction, hot flashes, loss of muscle mass, urinary incontinence, induced metabolic syndrome, loss of 
bone, and even cognitive impairment (McDowell et al., 2009). Bungay and Cappello (2009) reported urinary symptoms, such as incontinence and increased frequency, were very common because of prostate cancer itself and subsequent treatment. Additionally, increased frequency of urination at night and night sweats disrupted many men’s sleep and led to men feeling fatigued. Moreover, men often needed to make lifestyle changes by planning trips around bathroom facilities and decreasing water intake, especially at night (Bungay \& Cappello, 2009). Rivers and colleagues (2010) reported quality of life related to sexual dysfunction was the highest concern for men diagnosed with prostate cancer, which was an even higher concern than survival from the disease.

Eldefrawy and colleagues (2013) discovered a large burden is placed on the United States health care system because prostate cancer is so prevalent. As the United States attempts to decrease unnecessary medical costs, it is important to know how much money has been spent in the past and who has been paying for health care. The majority of the health care money in the United States was spent on hospital services as an inpatient (33\%) and physician services as an outpatient (23\%) (Eldefrawy et al., 2013). The authors reported the government accounts for $45 \%$ of the cost, employer $36 \%$, and patients 15\%. Wray and colleagues (2009) reported a five-year survival rate of almost $100 \%$ when prostate cancer was diagnosed in the localized stage. Not only could men live longer, but money could also be saved by the patients, insurance companies, and employers if screening for prostate cancer was completed and cancer was identified early in more easily treatable stages (Aubry et al., 2013). 


\section{Methodological Choices}

\section{Qualitative: Focus Group}

The use of focus groups is a qualitative, methodological approach designed to help researchers understand how people view and gain meaning from a specific subject matter. It is often used as an initial component of a research study (Doody, Slevin, \& Taggart, 2013). Participants are more likely to share opinions in a group when the members of the group are similar (Doody et al., 2013). Doody and colleagues (2013) found the group should consist of four to fourteen participants, so the group would not be too large where participants felt intimidated to share opinions, yet large enough to produce diversity of views. Both Friedman and colleagues (2012) and Carter and colleagues (2010) reported using audio-recordings of the focus group discussions helped researchers more accurately obtain information. Friedman and colleagues (2012) also found using open-ended questions with one moderator and one note taker was the best way to conduct a focus group. A focus group was utilized as the first piece of this study to validate the understanding of the survey among the target population and gain some very preliminary ideas about their opinions on prostate cancer.

\section{Quantitative: Survey}

Surveys have been conducted in the past to understand knowledge and attitudes regarding prostate cancer and screening among men from various ethnicities. Shaw, Vivian, Orzech, Torres, and Armin (2012) studied prostate cancer screening attitudes and concluded relationships were highly influential on attitudes toward prostate cancer screening in a qualitative and quantitative four-year prospective study. Shaw and colleagues (2012) used mainly chart abstraction and self-reported surveys among White, 
African American, Vietnamese, and Latino patients $(n=297)$ from a community health center serving low-income and underserved patients.

Allen and colleagues (2011) conducted surveys examining prostate cancer knowledge; screening preferences, history, and decisions; decisional consistency; and decision self-efficacy in 12 worksites among men $(n=812)$ who were 45 years old or older and predominately (89\%) White, non-Hispanic. It was reported men of other ethnicities than White, non-Hispanic needed the most assistance with the informed decision-making process for prostate cancer screening. A sample of 1,089 men from various ethnicities, who were 40-74 years old, were surveyed using the Gesellschaft für Konsumforschung Custom Research, Limited Liability Company's Knowledge Panel ${ }^{\circledR}$ to assess if the men intended to follow the USPSTF's recommendation not to participate in a PSA test, or if they intended to participate in a PSA test (Squiers et al., 2013). Factors that were positively correlated with having a PSA test done were higher income, having had a PSA in the past two years, identifying as Black, and being somewhat to very worried concerning risk for prostate cancer diagnosis. Telephone surveys were distributed among non-Latino White, Latino, African American, and Asian men who were first-degree relatives of men who were diagnosed with prostate cancer (Glenn et al., 2012). The researchers found fewer than $50 \%$ of these 1,029 men had participated in a PSA test throughout the past year. Factors that increased screening were having fewer barriers, having a physician recommend screening, having previously completed a PSA test, and having a first-degree relative as his brother versus his father (in all ethnic groups besides African Americans). 
The use of surveys has also been implemented in studies involving African American men. Patel and colleagues (2010) conducted surveys in three urban cities in Meharry Community Networks Program in Tennessee among low-income African American men $(n=293)$. The researchers concluded information regarding predictors of and obstacles to screening should be included in educational interventions among African American men. During a health fair, 229 African American men, who were 18 years old or older, were surveyed (Mitchell, 2011). It was found that several ecological and social factors influence health behavior. The survey consisted of a few questionnaires compiled into a self-administered packet. Mitchell (2011) was successful in developing a survey by combining several existing surveys for a specific population. Carter and colleagues (2010) conducted pre-test and post-test surveys among African American men $(n=76)$ in rural Alabama and found African American men were reluctant to discuss personal health issues. An educational intervention increased screening participation by $48 \%$ and increased knowledge of prostate cancer by $85 \%$ in one year.

Studies conducted among Afro-Caribbean American men have also utilized surveys as a data collection method. A total of 143 Haitian American men were given surveys to look at the congruence of perceived susceptibility with actual risk, the relationship between fear and perceived susceptibility, and how fear and perceived susceptibility correlate with screening behavior (Kleier, 2010). It was reported that perceived susceptibility was lower than actual risk, and perceived susceptibility was correlated with both fear and screening behavior, but fear was not correlated with screening behavior. The Powe Fatalism Inventory and the Personal Integrative Model of Prostate Cancer Disparity Survey were used to gather information from 211 United 
States-born and Caribbean-born Black men from 39-75 years old living in South Florida (Cobran et al., 2013). Place of birth and level of prostate cancer fatalism did not significantly influence PSA testing practice. 


\section{CHAPTER III \\ METHODOLOGY}

\section{Setting and Sample}

The setting for the study consisted of five churches in South Florida. The churches were selected through a convenience sample due to common involvement in the CHAMP organization. Based on the most recently published information on these churches, the demographic breakdown on ethnicity, excluding women and children, consisted of approximately 40\% African American and approximately 60\% AfroCaribbean American men (Parchment, 2004).

Preliminary data was obtained for this study from a focus group conducted at one of the participating churches. The sample consisted of eight African American and AfroCaribbean American men. All five churches were the setting for the distribution of the self-administered surveys. The surveys distributed included information on demographics, personal and family health history, prostate cancer knowledge, decisional conflict, perceived barriers, and spiritual well-being. Approximately 190 men from all five churches attend church on a regular basis, according to church leaders’ estimates. In this study, an African American man was defined as a man who identifies himself racially as Black, lives in the United States of America, and is of African descent. An Afro-Caribbean American man was defined as a man who identifies himself racially as Black, lives in the United States of America, and is of African and Caribbean descent, including, but not limited to Jamaican, Bahamian, Trinidadian, Haitian, and Guyanese. The inclusion criteria were men who self-identified as (a) African American or Afro-Caribbean American, even if they also self-identified as part of another ethnicity, 
(b) able to understand written and spoken English, (c) over 21 years old, (d) born a genetic male and still having at least part of his prostate (even if he had prostate surgery), and (e) willing to participate throughout the entire study. Exclusion criteria were men who self-identified as (a) not even partially African American or Afro-Caribbean American, (b) not able to understand written and spoken English, (c) under 21 years old, (d) not born a genetic male and not having at least a portion of his prostate, and (e) not able to participate throughout the entire study. Men had the option to refuse to participate or remain part of the study at any time.

The sample size for the surveys was calculated a priori. Considering a standard deviation of 1.6, as reported on a previous study that used the Prostate Cancer Screening Education (PROCASE) Knowledge Index (Hevey et al., 2009), this study was powered with a 95\% confidence interval of an estimated one-point width. Given the mean of 7.5 ( $S D=1.6)$ from Hevey and colleagues (2009), 40 participants were needed to estimate a 95\% confidence interval with a one-point width. Therefore, 40 African American men and 40 Afro-Caribbean American men were needed. Thus, if the observed mean was 7.5, then the $95 \%$ confidence interval of one-point width would range from 7.0 to 8.0, which would be fairly precise (Cohen, 1988).

The software GPower (version 3.1) was used post hoc to conduct power analyses. First, a power analysis was done for a two-tail t-test with a mean difference between two independent means (two groups). The effect size was large (0.8), and the alpha error probability was 0.05 for a power of 0.95 . Second, a power analysis was done for a chisquare test with a cross table analysis with a large effect size (0.5), and an alpha error probability of 0.05 for a power of 0.95 . 


\section{Procedures}

This study was a mixed methods study with a non-experimental design. The qualitative component consisted of conducting a focus group. The quantitative portion of the study entailed the completion of a survey packet (see Appendix A). Approval was obtained from the Florida International University Institutional Review Board (IRB) to undergo the proposed research study before recruitment or any data collection was conducted (see Appendix B).

The church leaders and Principal Investigator (PI) were able to recruit participants via word of mouth, verbal announcements, church bulletins, and posted flyers. Word of mouth was a recruitment strategy that was also successful in another study involving prostate cancer in African American men (Friedman et al., 2009). No prescreening occurred, but convenience sampling of men who were attending one of the churches was used to choose the study sample.

With both the focus group and the surveys, the PI verbally explained to the potential participants during or after each church meeting the purpose of the study, what would be asked of the participants during the study, the timeframe of the study, the risks and benefits of participation, and the voluntary nature of participation, along with ability to terminate without repercussions at any time. This information was also printed on the consent forms. Two copies of the consent forms were handed out to each participant. Participants read the consent forms; any questions were answered to their satisfaction; and they signed both copies prior to the start of the focus group or survey questionnaires. One copy was given to the participant, and the other copy was retained by the PI. After the participant signed the informed consent, the questionnaire packet was distributed. 
Upon completion of the survey packets, the participants returned the packets to the PI, who encouraged completeness of each packet and thanked each man for participating.

\section{Data Collection}

\section{Focus Group}

After the consent process and survey packets were completed, the focus group

discussion commenced, which lasted approximately 45 minutes. Although the purpose of the focus group was to validate understanding of the survey questions, four other prostate health topics were also discussed: health communication, knowledge, informed decision making, and barriers and beliefs. The PI, as the moderator, introduced each topic, and an assistant moderator managed the tape recorder and took written notes. All men who participated in the focus group were encouraged to share feelings and opinions freely. Following the focus group, the participants were provided with a free meal. The audio recording was transcribed verbatim into Microsoft Word ${ }^{\circledR}$.

\section{Surveys}

Participants for the surveys were recruited by church leaders and the PI via word of mouth, verbal announcements, church bulletins, and posted flyers at least one week prior to survey distribution. A verbal announcement was again made at the time of the survey distribution in each church meeting or service. Informed consent was signed before the surveys were delivered. The self-administered surveys were then distributed at all five churches. The surveys included six components: (1) demographic questions, (2) personal and family health history questions, (3) prostate knowledge questions, (4) informed decision-making questions as measured by men's level of conflict in the decision regarding prostate screening, (5) barriers to screening questions, and (6) beliefs 
regarding spiritual well-being questions. The PI collected all completed surveys during that same church meeting or service. No surveys were mailed to the PI or collected at a later date.

\section{Instruments}

Several instruments were combined into one questionnaire packet. Knowledge was assessed using a combined version of the Knowledge and Practice of Prostate Health Questionnaire and PROCASE Knowledge Index. Self-efficacy measured as conflict was reported from the Low Literacy Decisional Conflict Scale. Barriers were identified from a version of the Perceived Barriers Survey. Spiritual well-being was evaluated using the Functional Assessment of Chronic Illness Therapy-Spiritual Well-Being, a modified version for non-illness (FACIT-Sp Non-Illness).

General prostate health knowledge was measured by two questions from a modified version of the Knowledge and Practice of Prostate Health Questionnaire. It was important to examine men's understanding of the prostate before trying to examine their understanding of prostate cancer (Adesanya, Shittu, Awobajo, Otulana, \& Adesanya, 2013). Adesanya and colleagues (2013) tested internal consistency and reliability of the Knowledge and Practice of Prostate Health Questionnaire during a pilot study among ten participants from Nigeria. A test-retest method was used, with two weeks between testing sessions. Internal consistency and reliability of the instrument was evident with the Pearson correlation reported as 0.85 (Adesanya et al., 2013).

Prostate cancer knowledge was also measured by a 10-item knowledge index called the PROCASE Knowledge Index (Radosevich et al., 2004). In order to establish internal consistency reliability and validity, Radosevich and colleagues (2004) collected 
data via telephone interviews from a sample of 875 male veterans aged 50 and older, who were mainly White. The internal consistency reliability for the PROCASE Knowledge Index was found to be fair by Radosevich and colleagues (2004), with a KuderRichardson 20 (KR-20) value of 0.68. The validity of the PROCASE Knowledge Index was determined in three ways: content, construct, and criterion. The content was determined to be valid by the team of researchers during the developmental phase of the study. Construct validity was tested for convergent and discriminate validity. The convergent validity test indicated high PROCASE Knowledge Index scores were significantly associated with a history of exposure to PSA information, a history of abnormal PSA levels, and higher formal education. For discriminate validity, medication use and comorbidities demonstrated no statistically significant differences between low and high PROCASE Knowledge Index scores, except for chronic obstructive pulmonary disease. Criterion validity compared the results of the test with 29 expert health professionals from the Veterans Affairs (VA) Medical Center in Minneapolis who were from either urology or internal medicine services. These health professionals (physicians, registered nurses, and advanced practice nurses) completed a written version of the knowledge questions for comparison. A high percent agreement was found between the expert health professionals and all items of the PROCASE Knowledge Index questions with $D$-values all greater than 0.20 (Radosevich et al., 2004). The two scales combined to form this study’s Prostate Knowledge Scale, which consisted of 12 true or false questions. Four questions formed the symptoms subscale, and three questions were combined to produce the screening subscale. High knowledge for the total score and two 
subscale scores yielded a score greater than $70 \%$, and a low score was $70 \%$ or less (Adesanya et al., 2013; Radosevich et al., 2004).

Self-efficacy was measured by the level of decisional conflict concerning making an informed decision regarding prostate screening, as reported in the Low Literacy Decisional Conflict Scale. Self-efficacy is the level of confidence to which one feels a decision can be made and followed through, and it affects health behavior (Rosenstock et al., 1988). Low self-efficacy is also a factor for increasing decisional conflict (Allen et al., 2011). The Decisional Conflict Scale was chosen to measure self-efficacy because conflict and self-efficacy were found to affect each other in the informed decisionmaking process for prostate cancer screening by Allen and colleagues (2011). In addition, Lee and colleagues (2012) used the HBM to guide their study and reported on the relationship of self-efficacy's impact on screening behavior. The researchers found low self-efficacy was correlated with low participation in PSA testing among both African American and Afro-Caribbean American participants. The framework of the HBM was also used by Holt and colleagues (2009) to connect self-efficacy and decision making regarding prostate screening, and they concluded self-efficacy for screening was an essential part of making an informed decision to participate in prostate screenings.

The Low Literacy Decisional Conflict Scale was developed by Linder and colleagues (2011), with a total score and four subscale scores: uncertainty, informed, values, and support. The total score and all subscale scores from the Decisional Conflict Scale range from 0-100, with the higher the total score, the higher the level of decisional conflict (Linder et al., 2011). The items on the Decisional Conflict Scale have been 
interpreted as a score less than 25 is low conflict, 25-37.5 is moderate conflict, and greater than 37.5 is high conflict (O’Connor, 1995).

The Decisional Conflict Scale measured conflict in this study regarding prostate screening from the question, "Which do you prefer? Getting a PSA test, not getting a PSA test, or unsure.” The low literacy version was revised from the original 16-item Decisional Conflict Scale (O’Connor, 1995). The Decisional Conflict Scale was developed by O’Connor (1995) to assess to what degree a person feels informed, how uncertain someone is in his/her decision, and how much he/she perceives his/her decision is in keeping with his/her values. The Decisional Conflict Scale was tested among 909 participants regarding the decision concerning breast cancer screening or influenza immunization.

The Low Literacy Decisional Conflict Scale was used because it was designed to be simple for participants to understand and complete (Linder et al., 2011). Linder and colleagues (2011) conducted a randomized, controlled trial among 149 men from Texas with a mean age of 54. These men were Black (73.6\%), White (16.2\%), MexicanAmerican, Hispanic/Latino (8.8\%), and Other (1.4\%). The researchers reported a high discriminate validity regarding participation in prostate cancer screening using the Low Literacy Decisional Conflict Scale. The Low Literacy Decisional Conflict Scale had a fair construct validity except for the supported subscale, with most r's $>0.40$, and an excellent internal consistency and reliability (alphas $>0.80$ ). Taylor and colleagues (2006) reported a fairly good alpha reliability of 0.76 after two items were eliminated for the Decisional Conflict Scale among a sample of 238 African American men aged 40-70 from Washington, DC. These two items were confusing for the participants and were 
eliminated. The scale used in this study was comprised of 10 questions with a response format of “yes," "no,” and “unsure.”

Barriers were identified using a modified version of the Perceived Barriers Survey from the Cancer Awareness Survey called the Perceived Barriers Survey (Parchment, 2004). Eleven barriers were ranked from the greatest (being one) to the least (being eleven). Beliefs were examined in terms of spiritual well-being. Spiritual well-being was measured using the FACIT-Sp Non-Illness, which can be applicable to all men because it does not include a specific illness (Visser, Garssen, \& Vingerhoets, 2010). The survey has the last two questions modified to assess for beliefs related to difficult times, as opposed to chronic illness or prostate cancer. The questionnaire has 12 questions comprising three subscales: meaning, peace, and faith. Visser and colleagues (2010) found a relationship between high levels of spirituality and high levels of wellbeing in most studies and particularly in all 16 studies that used the FACIT-Sp version questionnaire. The original validation study for the FACIT-Sp was conducted among 1,617 participants, with 83.1\% being participants with cancer (Peterman, Fitchett, Brady, Hernandez, \& Cella, 2002). The median age was 54.6, a total of 53\% were female, and $47 \%$ were male. Internal consistency and reliability were high even among different cultures (Hispanic compared to White non-Hispanic compared to Black non-Hispanic). The Cronbach's alpha for the total scale was 0.87 , for the meaning/peace subscale was 0.81, and for the faith subscale was 0.88 (Peterman et al., 2002). Spirituality does not always consist of religion but does include seeking purpose and meaning in life (Holt et al., 2009). Visser and colleagues (2010) agreed meaning, peace, and faith were all components of spirituality. 


\section{Data Management and Analysis}

The data were managed using two systems called the Research Electronic Data Capture (REDCap) and the Statistical Package for the Social Sciences (SPSS). Twice the data were coded in REDCap, including assigning missing codes where questions were not answered. These codes were compared and adjustments were made as necessary. The data were entered separately and compared. The raw data were used to solve any differences between the two entries. The data were then exported into SPSS.

Consistent coding principles were used in managing the data to ensure accurate recording of the data. Data integrity was maintained throughout the various steps of the study: at collection, coding, and entry. Each participant was assigned a number as an identifier. Most questionnaires were completed. Although participants were encouraged to complete each question, some questions remained unanswered. The PI collected, managed, analyzed, and interpreted the data.

Descriptive statistics were computed, including frequency, mean, and standard deviation, for all independent and dependent variables separately. Independent variables included questions from the Demographic Questionnaire and the Personal and Family Health History Questionnaire. Demographic characteristics were assessed for each outcome to determine homogeneity in characteristics across outcome variables. Dependent variables included questions from the following questionnaires: the Prostate Knowledge Scale, the Decisional Conflict Scale, the Perceived Barriers Survey, and the FACIT-Sp Non-Illness scale (see Table C1 in Appendix C).

T-tests were used for the analysis of age, the Prostate Knowledge Scale, the Decisional Conflict Scale, and the FACIT-Sp Non-Illness scale for beliefs. Chi-square 
tests were used for the analysis of the demographics, except for age and for the Personal and Family Health History Questionnaire. A Mann-Whitney U Test was used for the Perceived Barriers Survey. Significance was measured at $\mathrm{p}<0.05$. Links were made among the theory of the HBM, research, and implications for practice.

Research Question 1: “Is there a relationship between ethnicity (African American and Afro-Caribbean American men) and level of prostate health knowledge?” Two questionnaires were examined to answer the first research question. The first questionnaire consisted of two questions from a modified version of the Knowledge and Practice of Prostate Health Questionnaire (Adesanya et al., 2013). The two questions investigated knowledge of prostate gland function and location, and had a score range from $0-2$ in a true or false format, with the correct response receiving one point and the incorrect response receiving no points for each question. A higher score indicated a higher level of general prostate knowledge.

The second set of questions was taken from the PROCASE Knowledge Index, with 10 questions and a score range of $0-10$, in a true or false format. A higher score indicated a higher level of prostate cancer and PSA knowledge. Both scores were analyzed together for a total score of 0-12. Two subscale scores, which only included seven of the total 12 questions, also were analyzed. The remaining five questions of the 12 were not part of a subscale score and were only analyzed as part of the total knowledge score. The knowledge of a screening subscale score was calculated using questions six, seven, and eight of the Prostate Knowledge Scale for a total score of 0-3. The knowledge of symptoms subscale score was calculated using questions three, four, nine, and ten of the Prostate Knowledge Scale for a total score of 0-4. For the total and 
two subscale scores, the higher the number of the score, the higher the prostate knowledge.

Research Question 2: “Is there a relationship between ethnicity (African American and Afro-Caribbean American men) and self-efficacy (measured by men’s level of conflict in making an informed decision regarding prostate screening)?” The second research question was answered by the use of the Low Literacy Decisional Conflict Scale. The scale contained 10 items in three response categories, with the format of the questions being yes (for a score value of 0), unsure (for a score value of 2), or no (for a score value of 4). The total score for the scale is determined by summing all values for each of the 10 questions, dividing by 10 , and multiplying by 25 . The score ranges from 0 (no decisional conflict) to 100 (extremely high decisional conflict). The four subcategories are uncertainty subscale score, which consists of two items (items 9 and 10) that are summed, divided by two, and multiplied by 25 for a score range from 0 (feels extremely certain of best choice) to 100 (feels extremely uncertain of best choice); informed subscale score, which consists of three items (items 1, 2 and 3) that are summed, divided by three, and multiplied by 25 for a score range from 0 (feels extremely informed) to 100 (feels extremely uninformed); values clarity subscale score, which consists of two items (items 4 and 5) that are summed, divided by two, and multiplied by 25 for a score range from 0 (feels extremely clear of personal values for benefits and risks/side effects) to 100 (feels extremely unclear of personal values); and support subscale score, which consists of three items (items 6, 7, and 8) that are summed, divided by three, and multiplied by 25 for a score range from 0 (feels extremely supported in 
decision making) to 100 (feels extremely unsupported in decision making) (O’Connor, 1995).

Research Question 3: “Is there a relationship between ethnicity (African American and Afro-Caribbean American men) and influencing factors on prostate screening behavior, including barriers to screening and beliefs measured by spiritual well-being?” Two scales were utilized to answer the third research question. One scale, the Perceived Barriers Survey, measured the level of perceived barriers regarding participation in a prostate screening by ranking each barrier. The Perceived Barriers Survey is a modified version of the Perceived Barriers Survey from the Cancer Awareness Survey, with the greatest barrier being number one, to the least barrier being number eleven. The score of each of the 11 barriers was averaged for African American and Afro-Caribbean American men. Then, it was determined which barrier was the greatest and which was the least.

The second scale was regarding spiritual well-being, which is called the FACITSp Non-Illness. The questionnaire has 12 questions containing three subscales: meaning (items 2, 3, 5, 8), peace (items 1, 4, 6, 7), and faith (items 9, 10, 11, 12), each with a score range of 0-16. The questionnaire uses a 5-point Likert-type scale, with each response having a point value assigned as either not at all (0), a little bit (1), somewhat (2), quite a bit (3), and very much (4) (Visser et al., 2010). After items 4 and 8 were reverse-coded, each subscale score was summed, multiplied by four, and then divided by the number of items answered. A total spirituality score was then derived by adding each of the three subscale scores to have a total score range of $0-48$. The direction of this scale is the higher the score, the higher the level of spiritual well-being. 


\section{Human Participants}

\section{Risks to Human Participants}

The participants were involved in a focus group or surveys. The age range was any age over 21 . Health status was not an inclusion or exclusion factor for this study. Special vulnerable populations, such as fetuses, neonates, pregnant women, children, prisoners, institutionalized individuals, or others who may be considered vulnerable populations, did not participate in this study.

No potential physical, financial, or legal risks to participants were apparent. The settings were churches that men regularly attended in order to provide men with a comfortable, familiar, and convenient environment for the study. Minimal risk was from time lost to participate in the focus group and survey questionnaires. The men participated during regularly scheduled church meetings and services, the survey packet was brief, and the questionnaire process was conducted privately to decrease participants' burden during the study.

Also, potential risks were emotional and psychological discomfort and risk related to the sensitive nature of asking questions on prostate health, especially questions regarding sexual function or dysfunction. This risk was minimal, and the PI was available to answer questions and resolve any concerns regarding the study and its questions. The risks to participants were reasonable in relation to the importance of the knowledge and benefits gained. No issues arose with the participants during the study.

\section{Potential Benefits to Human Participants and Others}

The most prominent potential benefit of the research was increased knowledge and understanding of African American and Afro-Caribbean American men's level of 
prostate health knowledge, level of conflict in the decision regarding prostate screening, and influencing factors on prostate screening behavior, including barriers to screenings and spiritual well-being. Another benefit was raising awareness in the African American and Afro-Caribbean American men who participated in the study, as evidenced by the focus group feedback. No physical or financial direct benefits were provided to participants in this study.

\section{Adequacy of Protection against Risks}

The signed informed consent documents were stored in a locked cabinet to protect against risks for this study. In addition, participants took part in the focus group anonymously or the surveys privately. Care was taken to have the PI available to answer any questions in a sensitive manner. Data were stored in a locked cabinet, and names of the participants were not available to anyone but the PI. Access to individually identifiable private information, including names of participants, was only available to the PI. The data were entered into REDCap and SPSS with identification numbers, not individual private identifiers. A password-protected computer was used for data entry. The PI established a monitoring plan for the overall framework of data and safety monitoring. The PI also was responsible for monitoring and reporting adverse events to the IRB, but none occurred. These actions were very effective protective measures and helped prevent and minimize potential risks, including risks to the privacy of individuals or confidentiality of data. 


\section{CHAPTER IV}

\section{RESULTS}

\section{Sample of Focus Group Participants}

The focus group consisted of men $(n=8)$ aged $40-70$ years old. The average age was 53.8 years old $(S D=10.3)$. For race, all self-identified as Black. For ethnicity, six self-identified as Afro-Caribbean American, one as African American, and one as "other" (not specified). All were married. For education, two reported having less than a high school education or General Educational Development (GED) completion, three had a high school education/GED completion, one had a bachelor's degree, and two had a postbaccalaureate education. Household annual income or salary was reported as one $\$ 20,000$ to less than $\$ 40,000$, one $\$ 40,000$ to less than $\$ 60,000$, three $\$ 60,000$ to less than $\$ 80,000$, two greater than $\$ 80,000$, and one did not respond. For health insurance coverage, five had private insurance, two had public, and one did not respond. For religious affiliation, two marked Protestant, four marked “other” (one Christian, one New Testament, one Pentecostal, and one only marked “other”), one had a double answer of Muslim and Mormon/LDS, and one did not mark anything.

\section{Focus Group Questionnaire Packet}

The focus group members were instructed to review the survey questions from the survey packet. Specifically, the wording used for the questionnaire packet was examined for validity of understanding and cultural relevance because various cultures have used different words to describe health issues, and words could have diverse meanings (Sobo, 2009). Every participant agreed all directions were clear and all questions helped raise awareness of prostate health. No suggestions were made to change anything in the 
packet before the surveys were to be conducted. Based on the participants' feedback from the focus group, the conclusion was the survey packet appeared to be clear and adequate. When asked concerning each questionnaire, the participants reiterated that the survey packet should be administered as it was presented to them. None thought any alterations, additions, or deletions were necessary. In fact, one gentleman said, "I actually liked, let me see, what is it? The Decisional Conflict Scale. I think that page really is an eye-opening page... I was going through it and was like whoa! The benefits, what options are available to you. Wow, I don't know a lot of stuff. It was good awareness." Therefore, because no recommendations were made to change the questions and all directions were clear, the questionnaire packet was administered without any alterations for the main quantitative survey portion of the study.

\section{Sample of Survey Participants}

A total sample of 113 men completed the surveys $(n=113)$. The characteristics of age, marital status, education, annual income, health insurance coverage, and religion for the survey sample are presented in Table 1. The participants' age range in the surveys was 23-93 years old with a mean age of 59.5 years old $(S D=16.4)$. Participants typically self-identified as Black ( $n=92,81.4 \%)$, were either African American ( $n=49$, 45.4\%) or Afro-Caribbean American ( $n=38,35.2 \%)$, were married ( $n=81,75.7 \%)$, had an associate's degree or some college or trade school $(n=36,34.3 \%)$, made $\$ 40,000$ to less than $\$ 60,000$ annually ( $n=28,29.8 \%$ ), had private insurance ( $n=59,56.7 \%$ ), and ( $n=41,36.3 \%$ ) marked "other” as religion. For religion, “other” was selected as Baptist ( $n=15,13.3 \%$ ), Christian/Christ/Jesus Christ/Christianity ( $n=12,10.6 \%)$, Pentecostal ( $n=4,3.5 \%)$, Church of God ( $n=3,2.7 \%)$, Episcopalian ( $n=3,2.7 \%)$, Anglican 
( $n=2,1.8 \%)$, and not specified ( $n=2,1.8 \%)$. For race, “other" was selected as Latin/Black ( $n=1,0.9 \%)$, Mixed $(n=1,0.9 \%)$, W.I. Black $(n=1,0.9 \%)$, and not specified ( $n=1,0.9 \%)$. For ethnicity, "other" was selected as not specified $(n=11$, 9.7\%), American ( $n=2,1.8 \%)$, African ( $n=1,0.9 \%)$, Irish/German/American ( $n=1$, $0.9 \%)$, Jamaican ( $n=1,0.9 \%)$, and Latin/Black $(n=1,0.9 \%)$.

The remaining results and discussion will be limited to African American and Afro-Caribbean American participants' responses, since this is the focus of this study. The characteristics of age, marital status, education, annual income, health insurance coverage, and religion for African American and Afro-Caribbean American participants are presented in Table 1. African American participants $(n=49)$ had a mean age of 53.4 $(S D=16.1)$. African American participants typically were married $(n=29,60.4 \%)$, had an associate's degree or some college or trade school $(n=16,34.0 \%)$, made $\$ 40,000$ to less than $\$ 60,000$ annually ( $n=13,31.7 \%)$, had private insurance $(n=28,59.6 \%)$, and indicated "other" for religion ( $n=27,58.7 \%)$. For religion, "other" for African American participants was selected as Baptist ( $n=15,30.6 \%)$, Christian/Christ/Christianity ( $n=8,16.3 \%$ ), not specified ( $n=2,4.1 \%)$, Episcopalian $(n=1,2.0 \%)$, and Pentecostal $(n=1,2.0 \%)$.

Afro-Caribbean American participants $(n=38)$ had a mean age of 61.2 $(S D=16.2)$. Afro-Caribbean American participants typically were married $(n=32$, 84.2\%), had an associate's degree or some college or trade school ( $n=12,31.6 \%)$, made $\$ 40,000$ to less than $\$ 60,000$ annually $(n=9,25.7 \%)$, had private insurance ( $n=25$, 67.6\%), and indicated Catholic for religion ( $n=17,44.7 \%)$. For religion, "other" for Afro-Caribbean American participants was selected as Pentecostal $(n=3,7.9 \%)$, 
Anglican ( $n=2,5.3 \%)$, Christian/Jesus Christ ( $n=2,5.3 \%)$, Church of God $(n=2$,

5.3\%), and Episcopalian ( $n=1,2.6 \%)$. Differences in age $(\mathrm{t}=-2.215, \mathrm{p}=0.030)$ and

religion $(\chi 2=14.082, \mathrm{p}=0.001)$ were observed to be statistically significant between

African American and Afro-Caribbean American participants.

Table 1

Characteristics of Participants

\begin{tabular}{|l|l|l|l|l|}
\hline Characteristics & $\begin{array}{l}\text { Total Sample } \\
(n=113)\end{array}$ & $\begin{array}{l}\text { African } \\
\text { American Men } \\
(n=49)\end{array}$ & $\begin{array}{l}\text { Afro- } \\
\text { Caribbean } \\
\text { American } \\
\text { Men }(n=38)\end{array}$ & Statistics \\
\hline Mean Age (SD) $^{\text {a }}$ & $59.5(16.4)$ & $53.4(16.1)$ & $61.2(16.2)$ & $t=-2.21^{*}$ \\
\hline Race $^{\text {b }}$ & $\%$ & $\%$ & $\%$ & \\
\hline Black & $81.4 \%$ & $100.0 \%$ & $100.0 \%$ & \\
\hline Caucasian & $4.4 \%$ & $0.0 \%$ & $0.0 \%$ & \\
\hline Latino & $2.7 \%$ & $0.0 \%$ & $0.0 \%$ & \\
\hline $\begin{array}{l}\text { Asian/Pacific } \\
\text { Islander }\end{array}$ & $2.7 \%$ & $0.0 \%$ & $0.0 \%$ & \\
\hline Other & $3.5 \%$ & $0.0 \%$ & $0.0 \%$ & \\
\hline Ethnicity & $\%$ & $\%$ & $\%$ & \\
\hline African American & $43.4 \%$ & $100.0 \%$ & $0.0 \%$ & \\
\hline $\begin{array}{l}\text { Afro-Caribbean } \\
\text { American }\end{array}$ & $33.6 \%$ & $0.0 \%$ & $100.0 \%$ & \\
\hline Hispanic & $3.5 \%$ & $0.0 \%$ & $0.0 \%$ & \\
\hline Other & $15.0 \%$ & $0.0 \%$ & $0.0 \%$ & \\
\hline Marital Status & $\%$ & $\%$ & $\%$ & \\
\hline Married & $71.7 \%$ & $59.2 \%$ & $84.2 \%$ & \\
\hline Single & $15.0 \%$ & $26.5 \%$ & $10.5 \%$ & \\
\hline Divorced & $5.3 \%$ & $8.2 \%$ & $5.3 \%$ & \\
\hline Partnered & $1.8 \%$ & $4.1 \%$ & $0.0 \%$ & \\
\hline Widower & $0.9 \%$ & $0.0 \%$ & $0.0 \%$ & \\
\hline Education & $\%$ & $0.0 \%$ & $5.3 \%$ & \\
\hline $\begin{array}{l}\text { Less than high school } \\
\text { /GED }\end{array}$ & $1.8 \%$ & $20.4 \%$ & $10.5 \%$ & \\
\hline High school/GED & $21.2 \%$ & $32.7 \%$ & & \\
\hline $\begin{array}{l}\text { Associate's degree or } \\
\text { some college or trade } \\
\text { school }\end{array}$ & $31.9 \%$ & & & \\
\hline Bachelor's degree & $18.6 \%$ & $24.5 \%$ & & \\
\hline Post-baccalaureate & $9.5 \%$ & & & \\
\hline & & & & \\
\hline
\end{tabular}




\begin{tabular}{|c|c|c|c|c|}
\hline education & & & & \\
\hline Income $^{\mathrm{a}}$ & $\%$ & $\%$ & $\%$ & $\chi^{2}=1.66$ \\
\hline Less than $\$ 20,000$ & $9.7 \%$ & $8.2 \%$ & $14.3 \%$ & \\
\hline $\begin{array}{c}\$ 20,000 \text { to less than } \\
\$ 40,000\end{array}$ & $13.3 \%$ & $10.2 \%$ & $20.0 \%$ & \\
\hline $\begin{array}{l}\$ 40,000 \text { to less than } \\
\$ 60,000\end{array}$ & $24.8 \%$ & $26.5 \%$ & $25.7 \%$ & \\
\hline $\begin{array}{l}\$ 60,000 \text { to less than } \\
\$ 80,000\end{array}$ & $15.9 \%$ & $16.3 \%$ & $20.0 \%$ & \\
\hline Greater than $\$ 80,000$ & $19.5 \%$ & $22.4 \%$ & $20.0 \%$ & \\
\hline Health Insurance ${ }^{\mathrm{a}}$ & $\%$ & $\%$ & $\%$ & $\chi^{2}=0.71$ \\
\hline Private & $52.2 \%$ & $57.1 \%$ & $67.6 \%$ & \\
\hline Public & $27.4 \%$ & $26.5 \%$ & $24.3 \%$ & \\
\hline None & $8.0 \%$ & $8.2 \%$ & $5.4 \%$ & \\
\hline Private and Public & $4.4 \%$ & $4.1 \%$ & $2.7 \%$ & \\
\hline Religion $^{c}$ & $\%$ & $\%$ & $\%$ & $\begin{array}{l}\chi^{2}= \\
14.08^{*}\end{array}$ \\
\hline Other & $36.3 \%$ & $55.1 \%$ & $26.3 \%$ & \\
\hline Catholic & $31.0 \%$ & $10.2 \%$ & $44.7 \%$ & \\
\hline Protestant & $25.7 \%$ & $28.6 \%$ & $28.9 \%$ & \\
\hline \multicolumn{5}{|c|}{$\begin{array}{l}\text { Note. } n \text { 's ranged from } 94-108 \text { for the Total Sample, } 47-48 \text { for African American men } \\
\text { and } 36-38 \text { for Afro-Caribbean American men due to missing data. } \\
\text { anot all participants from all ethnicities answered the question. }{ }^{b} \text { not all participants from } \\
\text { the Total Sample answered the question. }{ }^{c} \text { not all African American and Total Sample } \\
\text { participants answered the question. } \\
{ }^{*} \text { p }<0.05 \text {. }\end{array}$} \\
\hline
\end{tabular}

The Personal and Family Health History Questionnaire’s results are displayed in Table 2. Several African American participants ( $n=22,45.8 \%$ ) and Afro-Caribbean American participants $(n=14,37.8 \%)$ reported having a family history of prostate cancer or not knowing if they have a family history of prostate cancer. Also, some African American participants ( $n=12,25.5 \%)$ and Afro-Caribbean American participants ( $n=6,16.7 \%)$ reported not having a doctor check the prostate, or not knowing if a doctor had checked the prostate. In addition, almost half of African American participants ( $n=23,47.9 \%)$ and nearly a third of Afro-Caribbean American participants ( $n=11,29 \%$ ) did not have a PSA blood test done, or did not know if one 
was conducted. Furthermore, approximately one-third of African American participants ( $n=16,33.3 \%)$ and Afro-Caribbean American participants $(n=11,28.9 \%)$ reported having had urinary problems of difficulty urinating, pain on urination, frequent urination, blood in the urine, or not knowing if they experienced such urinary problems. No statistically significant differences were observed in the personal and family history questionnaire responses between African American and Afro-Caribbean American participants.

\begin{tabular}{|c|c|c|c|}
\hline Questions/Responses & $\begin{array}{l}\text { African } \\
\text { American } \\
\text { Men }(n=49)\end{array}$ & $\begin{array}{l}\text { Afro-Caribbean } \\
\text { American } \\
\text { Men }(n=38)\end{array}$ & Statistics \\
\hline $\begin{array}{l}\text { 1. History of prostate cancer in } \\
\text { your family }^{\mathrm{a}}\end{array}$ & & & $\chi^{2}=4.16$ \\
\hline Yes & $25 \%$ & $32.4 \%$ & \\
\hline No & $54.2 \%$ & $62.2 \%$ & \\
\hline I don’t know & $20.8 \%$ & $5.4 \%$ & \\
\hline $\begin{array}{l}\text { 2. Doctor ever checked your } \\
\text { prostate }^{\text {a }}\end{array}$ & & & $\chi 2=2.86$ \\
\hline Yes & $74.5 \%$ & $83.3 \%$ & \\
\hline No & $25.5 \%$ & $13.9 \%$ & \\
\hline I don't know & $0 \%$ & $2.8 \%$ & \\
\hline 3. Ever had a PSA ${ }^{\text {b }}$ & & & $\chi 2=3.59$ \\
\hline Yes & $52.1 \%$ & $71.1 \%$ & \\
\hline No & $39.6 \%$ & $21.1 \%$ & \\
\hline I don't know & $8.3 \%$ & $7.9 \%$ & \\
\hline 4. Ever had urinary problems ${ }^{\mathrm{b}}$ & & & $\chi 2=1.26$ \\
\hline Yes & $25 \%$ & $26.3 \%$ & \\
\hline No & $66.7 \%$ & $71.1 \%$ & \\
\hline I don't know & $8.3 \%$ & $2.6 \%$ & \\
\hline \multicolumn{4}{|c|}{$\begin{array}{l}\text { Note. n's ranged from } 47-48 \text { for African American men and } 36-38 \text { for Afro-Caribbean } \\
\text { American men due to missing data. } \\
\text { anot all participants from both ethnicities answered the question. }{ }^{\text {b }} \text { not all African } \\
\text { American participants answered the question. }\end{array}$} \\
\hline
\end{tabular}




\section{Prostate Knowledge}

The Prostate Knowledge Scale had a total knowledge score with subscale scores in knowledge of screening and knowledge of symptoms. These results are reported in Table 3. The total knowledge score had a range from 0-12, the knowledge of screening subscale score had a range from 0-4, and the knowledge of symptoms subscale score had a range from $0-3$. The higher the score, the higher the level of prostate knowledge for the total score and both subscale scores. High knowledge was determined to be a score greater than $70 \%$, and a low score was $70 \%$ or less for the total, as well as the subscale scores (Adesanya et al., 2013; Radosevich et al., 2004).

Both African American participants ( $n=49,8.3$ ) and Afro-Caribbean American participants ( $n=29,8.2)$ had a low total score. Also, both African American participants ( $n=46,2.7)$ and Afro-Caribbean American participants $(n=34,2.8)$ had a low knowledge of symptoms subscale score. However, African American participants $(n=47,2.4)$ and Afro-Caribbean American participants $(n=35,2.3)$ had a high knowledge of screening subscale score.

The symptom subscale score contained both the lowest correctly answered and highest correctly answered item. Only 33.3\% $(n=16)$ of African American participants and 27.0\% $(n=10)$ of Afro-Caribbean American participants answered item 3 ("Prostate cancer is the most common cause of problems with urination.") correctly, which was the lowest score out of any question. The question both African American participants ( $n=45,93.8 \%$ ) and Afro-Caribbean American participants ( $n=34,91.7 \%)$ answered correctly the most was item 4 ("Prostate cancer never causes problems with urination."). The differences observed were not statistically significant for the total knowledge scale 
score $(t=0.221, p=0.826)$, symptoms subscale score $(t=-0.595, p=0.554)$, or screening subscale score $(\mathrm{t}=1.155, \mathrm{p}=0.252)$ between African American and AfroCaribbean American participants.

\begin{tabular}{|c|c|c|c|}
\hline \multicolumn{4}{|c|}{$\begin{array}{l}\text { Table } 3 \\
\text { Prostate Knowledge Scale }\end{array}$} \\
\hline Scale/Subscales & $\begin{array}{l}\text { African } \\
\text { American Men } \\
M(S D) \\
(n=49)\end{array}$ & $\begin{array}{l}\text { Afro-Caribbean } \\
\text { American Men } \\
M(S D) \\
(n=38)\end{array}$ & Statistics \\
\hline $\begin{array}{l}\text { Total }^{\mathrm{a}} \\
\text { (items 1-12) }\end{array}$ & $8.3(1.4)$ & $8.2(2.0)$ & $\mathrm{t}=0.22$ \\
\hline $\begin{array}{l}\text { Symptoms }^{\mathrm{a}} \\
\text { (items 3, 4, 9, 10) }\end{array}$ & $2.7(0.8)$ & $2.8(0.9)$ & $t=-0.60$ \\
\hline $\begin{array}{l}\text { Screening }^{\mathrm{a}} \\
\text { (items 6, 7, 8) }\end{array}$ & $2.4(0.6)$ & $2.3(0.5)$ & $t=1.16$ \\
\hline \multicolumn{4}{|c|}{$\begin{array}{l}\text { Note. The total score ranges from } 0-12 \text {. The symptoms subscale score ranges from } 0 \text { - } \\
\text { 4. The screening subscale score ranges from } 0-3 \text {. A higher score means a higher level } \\
\text { of prostate knowledge for the total score and both subscale scores. n's ranged from } 41 \text { - } \\
47 \text { for African American men and } 29-35 \text { for Afro-Caribbean American men due to } \\
\text { missing data. } \\
\text { anot all participants from both ethnicities answered the question. }\end{array}$} \\
\hline
\end{tabular}

\section{Decisional Conflict}

Self-efficacy was measured by men’s level of conflict in making an informed decision regarding prostate screening using the Decisional Conflict Scale. The Decisional Conflict Scale has a total score and four subscale scores: uncertainty, informed, values, and support. These scores are depicted in Table 4. The Decisional Conflict Scale examined the conflict from the question, "Which do you prefer? Getting a PSA test, not getting a PSA test, or unsure.” Seven African American participants (14.9\%) and eight Afro-Caribbean American participants (22.2\%) preferred not getting a PSA test or unsure. The total score and all subscale scores from the Decisional Conflict Scale ranged from 0-100. The higher the total score, the higher the level of decisional 
conflict (Linder et al., 2011). Both African American participants $(n=45,36.0)$ and Afro-Caribbean American participants $(n=31,28.2)$ had moderate total decisional conflict (O’Connor, 1995). The higher the uncertainty subscale score, the higher the uncertainty of the choice. African American participants ( $n=47,36.2)$ and AfroCaribbean American participants ( $n=37,29.1)$ had moderate uncertainty regarding best choice. The higher the informed subscale score, the greater the participant feels uninformed. African American participants $(n=47,41.1)$ felt highly uninformed and Afro-Caribbean American participants $(n=36,32.9)$ felt moderately uninformed of the best choice. The higher the values subscale score, the greater the participant feels unclear of personal values. African American participants $(n=47,46.3)$ felt highly unclear and Afro-Caribbean American participants $(n=36,31.9)$ felt moderately unclear concerning personal values for benefits and risks/side effects of the best choice. The higher the support subscale score, the greater the participant feels unsupported in decision making. African American participants $(n=45,23.3)$ and Afro-Caribbean American participants $(n=33,23.2)$ felt highly supported in the decision-making process. The differences observed for total $(t=1.178, p=0.243)$, uncertainty $(t=0.882, p=0.380)$, informed $(t=1.070, p=0.288)$, values $(t=1.659, p=0.101)$, and support $(t=0.017, p=0.987)$ scores were not statistically significant between African American and Afro-Caribbean American participants.

\begin{tabular}{|l|l|l|l|}
\hline $\begin{array}{l}\text { Table } 4 \\
\text { Decisional Conflict Scale }\end{array}$ \\
\hline Scale/Subscales & African & Afro-Caribbean & Statistics \\
& American Men & American Men & \\
& $M(S D)$ & $M(S D)$ & \\
& $(n=49)$ & $(n=38)$ & \\
\hline Total $^{\mathrm{a}}$ & $36.0(29.6)$ & $28.2(26.2)$ & $\mathrm{t}=1.18$ \\
\hline
\end{tabular}




\begin{tabular}{|c|c|c|c|}
\hline (items 1-10) & & & \\
\hline $\begin{array}{l}\text { Uncertainty }^{\mathrm{a}} \\
\text { (items } 9,10)\end{array}$ & $36.2(36.4)$ & $29.1(37.0)$ & $t=0.88$ \\
\hline $\begin{array}{l}\text { Informed }^{\mathrm{a}} \\
\text { (items 1, 2, 3) }\end{array}$ & $41.1(37.2)$ & $32.9(31.5)$ & $\mathrm{t}=1.07$ \\
\hline $\begin{array}{l}\text { Values }^{\mathrm{a}} \\
\text { (items 4, 5) }\end{array}$ & $46.3(41.4)$ & $31.9(35.7)$ & $t=1.66$ \\
\hline $\begin{array}{l}\text { Support }^{\mathrm{a}} \\
\text { (items 6, 7, 8) }\end{array}$ & $23.3(24.2)$ & $23.2(28.2)$ & $t=0.02$ \\
\hline \multicolumn{4}{|c|}{$\begin{array}{l}\text { Note. All scores range from 0-100. The higher the total score, the higher the level of } \\
\text { decisional conflict. The higher the uncertainty subscale score, the higher the } \\
\text { uncertainty of the choice. The higher the informed subscale score, the greater the } \\
\text { participant feels uninformed. The higher the values subscale score, the greater the } \\
\text { participant feels unclear of personal values. The higher the support subscale score, the } \\
\text { greater the participant feels unsupported in decision making. n's ranged from } 45-47 \text { for } \\
\text { African American men and 31-37 for Afro-Caribbean American men due to missing } \\
\text { data. } \\
\text { anot all participants from both ethnicities answered the question. }\end{array}$} \\
\hline
\end{tabular}

\section{Barriers}

The Perceived Barriers Survey, a modified version of the Perceived Barriers Survey from the Cancer Awareness Survey, ranked barriers from the greatest barrier being number one to the least barrier being number eleven. The results are presented in Table 5. The greatest barrier (ranked as a 1 or 2) for African American participants was “Can’t afford it” and "Doctor did not tell me I needed it,” with a count of 12 (24.5\%).

The greatest barrier (ranked as a 1 or 2) for Afro-Caribbean American participants was "Exam is embarrassing," with a count of 9 (23.7\%). The differences in barriers observed were not statistically significant between African American and Afro-Caribbean American participants using A Mann-Whitney U Test.

\section{Table 5}

Barriers Ranked as One or Two for Prostate Cancer Screening

\begin{tabular}{|l|l|} 
African American Men $(n=49)$ & $\begin{array}{l}\text { Afro-Caribbean American Men } \\
(n=38)\end{array}$ \\
\hline
\end{tabular}




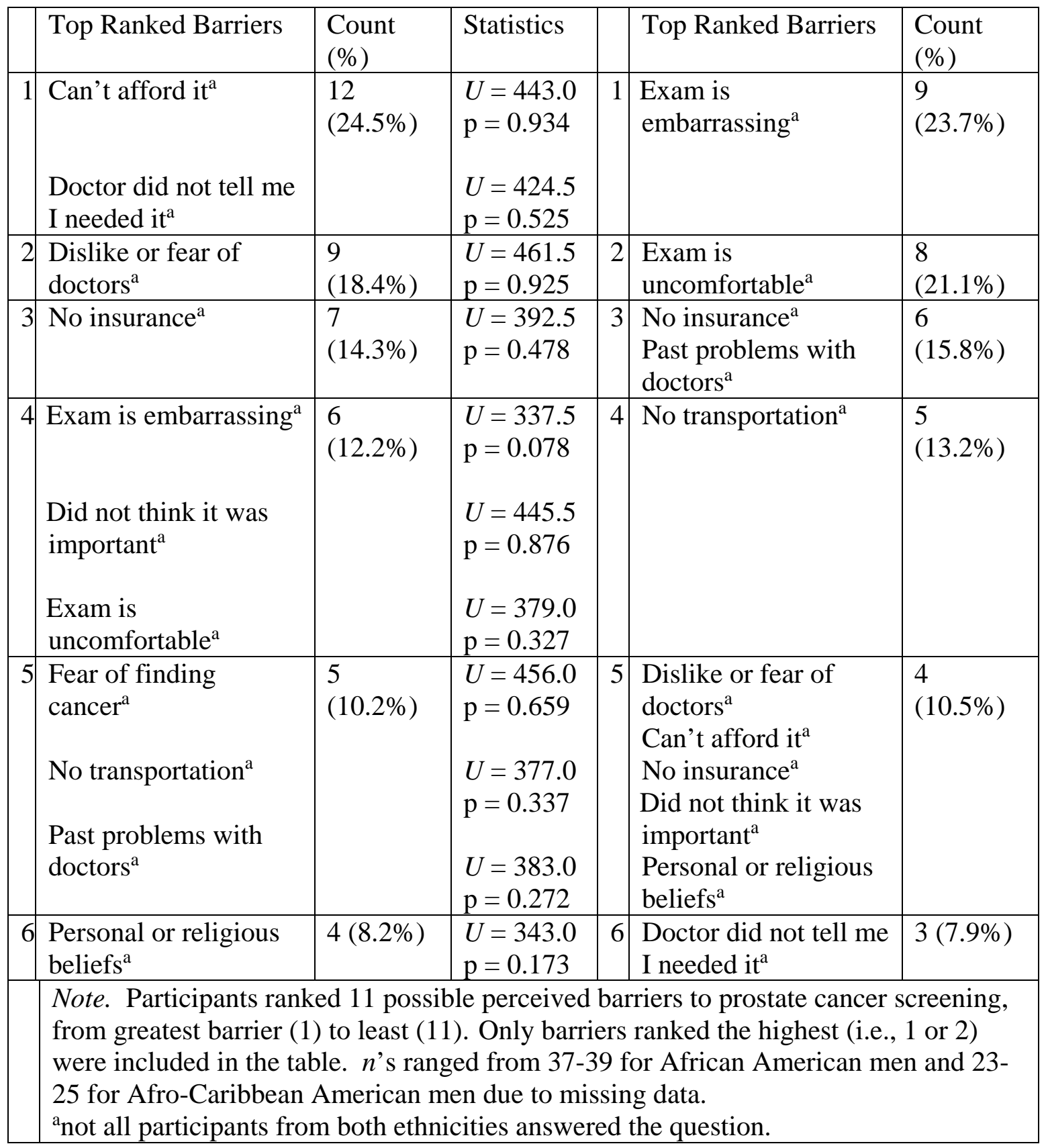

\section{Beliefs}

The FACIT-Sp Non-Illness scale to measure spiritual well-being had 12 questions comprised of three subscales: meaning, peace, and faith. The results are displayed in Table 6. Each subscale had a score range of 0-16. When combined a total spirituality 
score with a range of 0-48 was obtained. The higher the score, the higher the reported level of spiritual well-being (Visser et al., 2010). Both African American participants ( $n=41,42.6)$ and Afro-Caribbean American participants $(n=36,41.9)$ had high total spiritual well-being. Also, African American participants $(n=44,14.6)$ and AfroCaribbean American participants $(n=37,14.2)$ had high levels of meaning in life. In addition, African American participants $(n=45,13.3)$ and Afro-Caribbean American participants ( $n=38,13.0)$ had high levels of peace in life. Furthermore, African American participants $(n=41,14.7)$ and Afro-Caribbean American participants $(n=37$, 14.5) had high levels of faith in life. The differences observed for total $(\mathrm{t}=0.442$, $\mathrm{p}=0.660)$, meaning $(\mathrm{t}=0.758, \mathrm{p}=0.451)$, peace $(\mathrm{t}=0.481, \mathrm{p}=0.632)$, and faith $(\mathrm{t}=0.449, \mathrm{p}=0.654)$ scores were not statistically significant between African American and Afro-Caribbean American participants.

\begin{tabular}{|c|c|c|c|}
\hline \multicolumn{4}{|c|}{$\begin{array}{l}\text { Table } 6 \\
\text { FACIT-Sp Non-Illness Scale }\end{array}$} \\
\hline Scale/Subscales & $\begin{array}{l}\text { African American } \\
\text { Men } \\
M(S D) \\
(n=49)\end{array}$ & $\begin{array}{l}\text { Afro-Caribbean } \\
\text { American Men } \\
M(S D) \\
(n=38)\end{array}$ & Statistics \\
\hline $\begin{array}{l}\text { Total }^{\mathrm{a}} \\
\text { (items 1-12) }\end{array}$ & $42.6(4.9)$ & $41.9(7.8)$ & $t=0.44$ \\
\hline $\begin{array}{l}\text { Meaning }{ }^{\mathrm{a}} \\
\text { (items 2, 3, 5, 8) }\end{array}$ & $14.6(1.7)$ & $14.2(3.0)$ & $t=0.76$ \\
\hline $\begin{array}{l}\text { Peace }^{\text {b }} \\
\text { (items 1, 4, 6, 7) }\end{array}$ & $13.3(2.8)$ & $13.0(2.6)$ & $t=0.48$ \\
\hline $\begin{array}{l}\text { Faith }^{\mathrm{a}} \\
\text { (items 9, 10, 11, 12) }\end{array}$ & $14.7(1.9)$ & $14.5(2.9)$ & $t=0.45$ \\
\hline \multicolumn{4}{|c|}{ 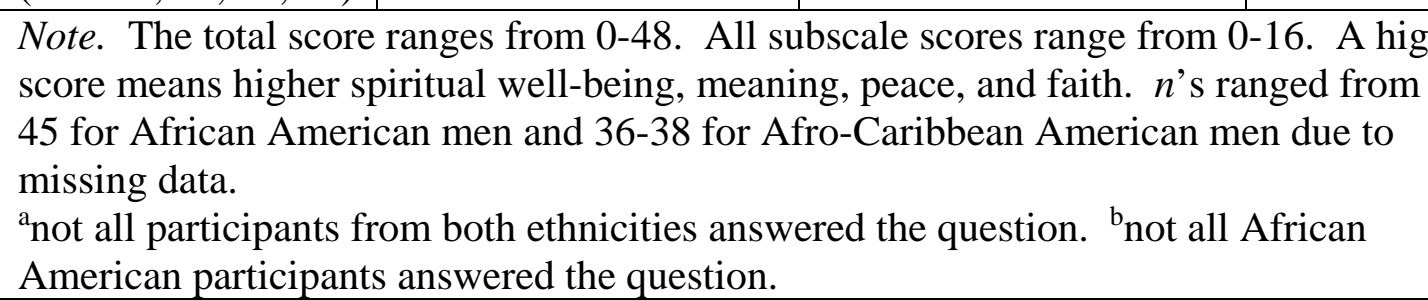 } \\
\hline
\end{tabular}




\section{Summary}

In this population at high risk for aggressive prostate cancer, knowledge about prostate health and prostate cancer screening participation was low. Likewise, African American participants felt highly uninformed and unclear of the personal values of benefits, risks, and side effects that matter most, as related to PSA testing, yet AfroCaribbean American participants felt only moderately uninformed and only moderately unclear of personal values. Although both African American and Afro-Caribbean American men felt only moderate overall conflict and uncertainty in their decision and highly supported in the decision-making process, lack of conflict, certainty in the decision, and support were insufficient to increase PSA test participation. The greatest barrier for African American participants was a tie between "Doctor did not tell me I needed it” and “Can’t afford it," and for Afro-Caribbean American participants it was "Exam is embarrassing." For the FACIT-Sp Non-Illness, both ethnicities had high scores of spiritual well-being in all scales. 


\section{CHAPTER V}

\section{DISCUSSION}

This chapter discusses the study findings, study limitations, implications, suggested future research to be conducted based on the findings, and a summary of the study. All findings are discussed in relation to the HBM and other literature on prostate health among African American and Afro-Caribbean American men. The study purpose was to assess the likelihood of action for and factors influencing choice regarding prostate cancer screenings. It was also to discover if a relationship existed between ethnicity (African American and Afro-Caribbean American men) and knowledge of prostate health, self-efficacy (measured by men's level of conflict in making an informed decision regarding prostate screening), perceived barriers to prostate screening, and beliefs measured by spiritual well-being. The findings showed a difference between ethnicity and perceived barriers of prostate cancer screening, and a similarity between ethnicity and the level of prostate health knowledge, spiritual well-being, and informed decision making regarding prostate screening.

This study has helped fill part of the knowledge gap associated with these ethnic groups at high risk for early-onset and aggressive prostate cancer (Anderson et al., 2013) by examining results separately and comparing between the two groups. Knowledge levels, self-efficacy, barriers, and beliefs regarding prostate health reported from African American and Afro-Caribbean American participants in this study revealed several similarities and few differences between the two ethnic groups. Both the finding of similarities and differences provides guidance for educators and researchers when 
addressing the problem of low routine prostate cancer screening rates in a high-risk population.

\section{Barriers}

African American participants reported barriers to screening differently than Afro-Caribbean American participants in this study. The theoretical construct of the HBM examined was perceived barriers within both ethnicities. One component of the HBM indicates a person will take a health-related action if the person feels perceived benefits outweigh perceived barriers (Lee et al., 2012). Also, while using the HBM as a framework in a study conducted among a sample of predominantly African American men, high perceived barriers prevented or delayed the participation in prostate screenings (Oliver et al., 2011).

Several other studies reported perceived barriers among African American and Afro-Caribbean American men resulted in less participation in a PSA test, a DRE, or both (Consedine, 2012; Friedman et al., 2009; Lee et al., 2012; Odedina et al., 2009;

Parchment, 2004; Pedersen et al., 2012). Likewise, in this study, perceived barriers were identified and screening practices were reportedly low. From the Personal and Family Health History Questionnaire in this study, approximately half of the African American participants and one-third of the Afro-Caribbean American participants reported not knowing they had, or never having had, a PSA test. One-fourth of African American participants and one-sixth of Afro-Caribbean American participants reported not knowing they had, or never having had, a DRE. A potential target for intervention work in the future should be increasing patient knowledge regarding evidence-based screening practices. 
African American participants reported different barriers to screening than AfroCaribbean American participants. African American participants ranked structural or societal barriers highest on the Perceived Barriers Survey. The participants indicated the greatest barriers were “Can’t afford it,” "Doctor did not tell me I needed it,” and "Dislike or fear of doctors.” African American participants in the focus group also reported doctor-related barriers, including not receiving a recommendation from the doctor for prostate cancer screening and overall mistrust of doctors. Afro-Caribbean American participants ranked personal barriers regarding the screening exam highest: "Exam is embarrassing” and "Exam is uncomfortable.” Participants in the focus group who were Afro-Caribbean American also reported fear and pain from the exam as barriers.

These findings were consistent with the literature that African American men commonly reported barriers to screening as structural or societal, including lack of trust of the health care system, deficient communication with health care providers, misunderstanding between diagnostic tests and screening, inconvenient clinic hours, and limited access to health care services (Friedman et al., 2009). Personal barriers concerning the screening exam were reported by Afro-Caribbean American participants in this study. These findings were consistent with other studies of Afro-Caribbean American men who had fear (Kleier, 2010), anxiety and worry (Consedine, 2012), or concerns regarding privacy and participating in screening exams (Ng et al., 2013).

African American and Afro-Caribbean American participants identified different barriers for prostate screening. African American participants were more concerned with structural or societal barriers. However, Afro-Caribbean American participants were more concerned with personal barriers. 


\section{Prostate Knowledge}

Total knowledge and symptoms knowledge were low ( $70 \%$ or less) and screening knowledge was high (greater than 70\%) among both ethnicities. Knowledge was a theoretical construct of the HBM that this study examined. The HBM linked a low level of knowledge with decreased preventive health behavior for prostate screenings, according to Holt and colleagues (2009). The researchers concluded knowledge was an important component needed for informed decision making to participate in prostate screening. Likewise, low prostate cancer knowledge and low rates of screening have been observed throughout the United States among various ethnicities (Rivera-Ramos \& Buki, 2011). In this study, low total prostate knowledge and low screening rates were also reported.

When each question from the questionnaire was considered separately, a question on urinary symptoms (item 4 "Prostate cancer never causes problems with urination.”) was the question most participants from both ethnicities answered correctly. However, a question on urinary symptoms (item 3 "Prostate cancer is the most common cause of problems with urination.”) was also the question most participants from both ethnicities answered incorrectly, which revealed participants were unaware of a common symptom. There is typically a direct relationship between knowledge level and preventive health behavior practices (Rivera-Ramos \& Buki, 2011). Consistent with this association between knowledge and behavior, most study participants had a low level of knowledge and reported low screening behavior in relation to their risk of prostate cancer.

The items on the Prostate Knowledge Scale were interpreted as a high level of knowledge if the score was greater than $70 \%$ on the scale or subscale, and a low level of 
knowledge if lower than 70\% (Adesanya et al., 2013; Radosevich et al., 2004). Results were compared to related research on prostate health knowledge, and knowledge scores were expected to be low based on the low knowledge results among men of African descent from other studies (Odedina et al., 2011; Rivers et al., 2010). In addition, Odedina and colleagues (2011) stated a reason for prostate cancer disparities was low knowledge levels regarding prostate cancer among men of African descent.

In this study, both African American and Afro-Caribbean American participants demonstrated a low level of prostate health knowledge. Overall knowledge levels were consistent with previous research, but high levels of screening knowledge were observed. However, for this high-risk group, knowledge about screening was not associated with increased prostate cancer screening behavior, as evidenced by the fact that almost half of the African American and nearly a third of the Afro-Caribbean American participants did not have a PSA blood test done, or did not know if one was conducted. Although the study participants were knowledgeable about prostate screening, this did not result in seeking preventive health services.

\section{Beliefs}

Both African American and Afro-Caribbean American participants had high levels of spirituality. Although spiritual well-being is not a theoretical construct of the HBM, it has been related to other theoretical constructs, including knowledge and selfefficacy (Drake et al., 2010; Holt et al., 2009). Spiritual well-being was investigated in this study because all participants are members of a Christian church community; and a previous study found people who actively attended religious groups or services have higher spiritual well-being, as well as improved health, and lived longer than those who 
did not (Stojković \& Prlić, 2012). However, increased spirituality does not necessarily require church attendance (Holt et al., 2009). Spirituality has been observed to be positively correlated with well-being (Visser et al. 2010).

As found by Stojković and Prlić (2012), the majority of the study participants from both ethnicities showed high levels of spiritual well-being in this study.

Saunders and colleagues (2013) found the majority of African American men were highly involved in, and frequently attended, churches. This involvement could increase spiritual well-being and make churches successful settings for prostate health interventions (Stojković \& Prlić, 2012). In addition, using churches as settings has helped educate African American men (Drake et al., 2010; Holt et al., 2009; Jackson et al., 2014; Parchment, 2004) and Afro-Caribbean American men (Parchment, 2004) regarding prostate health issues and screening practices.

Community involvement in churches has been shown to increase the number of African American men willing to complete prostate screenings (Emerson et al., 2009; Holt et al., 2009). A church-based intervention was tested in predominantly African American churches and showed an increase in informed decision making, knowledge, and self-efficacy in terms of prostate screening (Drake et al., 2010). Holt and colleagues (2009) piloted a spiritually based educational intervention among African American men and found knowledge and self-efficacy for informed decision making in regards to prostate screening increased in a spiritually based group to a greater degree than the nonspiritually based group.

Churches were also the setting of this study, and all five churches were part of a faith-based and medical center partnership called CHAMP. The churches were the faith- 
based organizations and consisted of various Christian denominations throughout the community. The medical partner was Baptist Health System of South Florida, which is a faith-based medical organization guided by Christian values with a goal to improve the well-being of the community. The similar mission of the faith-based organizations and the medical partner strengthens their relationship and optimizes their goal of improving health in the South Florida community.

Partnerships between faith-based organizations and medical centers were studied by Kegler, Hall, and Kiser (2010), who found an overall use of resources increased and health disparities decreased in communities where faith-based and health organizations collaborated. Similarly, Levin (2014) reported these partnerships have been successful at providing health care, especially to underserved populations, and have helped decrease health disparities in the United States. These partnerships have expanded to the national level to produce federal faith-based resources that assist in accomplishing Healthy People 2020 goals, decreasing health disparities, and improving use of health resources across the nation (Levin, 2014). Therefore, faith-based and medical partnerships could be used more frequently and continued as a nationally led initiative. According to Levin (2014), this national initiative was started during the Clinton Administration, with the signing of the "charitable choice" doctrine in 1996. It was sustained with the development of the White House Office of Faith-Based and Community Initiatives by the Bush Administration in 2001. Subsequently, it continued through the Obama Administration, within the Department of Health and Human Services, and was renamed as the Office of Faith-Based and Neighborhood Partnerships (Levin, 2014). 


\section{Decisional Conflict}

Self-efficacy in this study was measured by men's level of conflict in making an informed decision regarding prostate screening using the Decisional Conflict Scale. African American participants felt highly uninformed and Afro-Caribbean American

participants felt moderately uninformed. However, both ethnicities felt highly supported in the decision-making process. Support from family and friends was an important factor, identified during the focus group, in reducing decisional conflict for both ethnicities.

Men of African descent having perceived high levels of support in their prostate screening decisions was consistent with the literature. Jones and colleagues (2010) reported African American men participated in prostate screenings when a friend and/or family member had prostate cancer and if friends and/or family were involved in the decision-making process. Participants in this study of active church members found perceived high levels of support for screening decisions. This is consistent with the findings of another study that reported African American men experience high levels of perceived support from church membership in making screening decisions (Holt, 2009). Although both African American and Afro-Caribbean American participants reported high levels of support and moderate total decisional conflict regarding intent to participate in a PSA testing, actual screening practices were reportedly low.

\section{Perceived Cost}

Perceived cost is a theoretical construct from the HBM. Although this study did not measure perceived cost specifically, perceived cost barriers to screening were measured. Interestingly, although approximately half (52.2\%) of participants reported 
having private health insurance and greater than one in four had public insurance (i.e., Medicare or Medicaid), the barrier of “Can’t afford it” was still ranked high among African American participants (tied for the highest-ranked barrier) and both ethnicities ranked "No insurance" (reported in $8 \%$ of participants) as the third greatest barrier. Previously, a lack of health insurance has been found to lead to men not participating in prostate screenings (Burns, Walsh, Sharp, \& O'Neill, 2012).

The Patient Protection and Affordable Care Act expanded insurance coverage to approximately 30 million Americans (Andrews et al., 2013), and the coinsurance and deductible payments were waived for PSA screenings through the Social Security Act. Therefore, the barrier of not being able to afford screening should see a reduction over time (Stranne, 2011). Burns and colleagues (2012) found men with private health insurance were more likely to participate in a PSA screening than men without it, and private health insurance coverage was the biggest factor in contributing to differences in screening practices among men. By increasing knowledge regarding coverage of insurance for prostate cancer screening, fewer men should view "financial cost" as a barrier for screening.

Still, other costs, in addition to the blood work and office visit exam involved with screenings, may have been factors influencing the perceived cost barrier reported in this study. Martin, Lord, Verry, Stockler, and Emery (2013) found some costs of screening were inconvenience of the screening, side effects from treating indolent tumors, and harm from biopsies. It is expected that with increased knowledge regarding both screening insurance coverage and cost of managing prostate cancer in low-risk 
stages versus high-risk stages among men from both ethnicities, the barrier of "Can’t afford it” should decrease and more men should participate in prostate screening.

\section{Age}

Age is an important characteristic to consider when planning prostate cancer education, and African American men had an improved response to education when materials were tailored to their age (Friedman et al., 2009). Both groups in this study had a mean age of over 45, and all participants in the study by Friedman and colleagues (2009) were 45 or older, so the approaches in education and recommendations would be comparable. Age was also a predictive variable for African American men participating in a prostate cancer screening (Lehto et al., 2010). Lehto and colleagues (2010) reported men 50 or older were more likely to participate in screenings than men younger than 50 . Again, both groups in this study had a mean age over 50, and Lehto and colleagues (2010) found a difference only between men younger than 50 and men aged 50 or older.

Younger men, including any man older than 21, could have participated in this study. Younger men were not excluded from this study because limited research regarding prostate cancer has been completed among men of African descent younger than 45 (Miller, 2014). Because the American Cancer Society (2015) recommends men of African descent to begin discussion with their health care provider regarding prostate cancer screening after 45 years of age, less research has been conducted among men younger than 45. However, earlier outreach to younger men is important in order to increase knowledge of screening and health values before men of African descent turn 45 and need to make a decision regarding prostate cancer screening (Lehto et al., 2010). 
The American Cancer Society (2015) reported 56\% of prostate cancer cases were diagnosed in men aged 65 or older and 97\% were diagnosed in men aged 50 or older, and the average age of diagnosis was approximately 66. The mean age for African American participants was 53.4 and for Afro-Caribbean American participants, it was 61.2 years old. There was a statistically significant difference in mean age in this study between the two groups. The importance of the difference in mean age was decreased because the mean ages for participants in both ethnic groups were older than 45 , which was the recommended age for men of African descent to start counseling with a health care provider concerning screening, as per the American Cancer Society (2015).

\section{Limitations}

Some limitations of this study were observed. First, the limitation of self-reported prostate screening participation without data gathered from medical records was noted (Polit, 2010; Waltz, Strickland, \& Lenz, 2010). It is possible participants were involved in PSA and DRE testing without being aware. Not being aware if testing had occurred is problematic, as the individual would not know when to seek further screening and how often these appointments should occur.

Second, data regarding the participants’ preference to participate in PSA testing was collected, but not DRE testing. Decisional conflict regarding PSA testing could have been different from the decisional conflict regarding DRE testing. The DRE test is an invasive test performed by a health care provider, who checks for abnormalities by palpating the prostate through introduction of a finger into the rectum (Wray et al., 2009). Also, men reportedly have not participated in DRE testing because they felt embarrassed to get an exam and lacked knowledge of the screening procedure (Ferrant et al., 2011). 
Differences in preference to participate in a DRE versus a PSA test could be due to the nature of the DRE exam, personal factors, and lack of knowledge regarding the DRE exam itself (Ferrant et al., 2011; Wray et al., 2009).

Finally, the study only sampled participants using convenience sampling from participants who were easily accessible to the PI. Only one network of churches in a single region was used for recruiting participants, and men were not queried about their country of origin and length of time living in the United States. Therefore, these findings have uncertain generalizability to men from other religious organizations and various settings (Polit, 2010; Waltz et al., 2010). Men from different countries of origin with various levels of acculturation from living in the United States could have differing knowledge and beliefs regarding prostate health. However, these findings are consistent with other studies. Rivera-Ramos and Buki (2011) also reported low prostate cancer knowledge among men of various ethnicities throughout the United States. In addition, other researchers reported similar barriers and beliefs regarding prostate screening practices, including lack of trust of the health care system, deficient communication with health care providers, misunderstanding between diagnostic tests and screening, (Friedman et al., 2009), fear of screening (Kleier, 2010), and anxiety and worry concerning screening (Consedine, 2012).

\section{Implications}

This study was able to fill some gaps in the current literature on prostate health knowledge, self-efficacy in screening decision making, perceived barriers regarding prostate screening, and spiritual well-being as related to the theoretical constructs of the HBM among African American and Afro-Caribbean American men. Findings 
contributed to the body of knowledge by noting decreased screening practices were related to issues concerning prostate cancer screening, lack of knowledge, and past negative experiences. The implications of these findings could be categorized into three main areas: patient education, faith-based and medical partnerships, and improved nursing practice.

First, data from this study indicating a disparity between high levels of prostate screening knowledge and low screening behavior could be used in conjunction with other studies to determine further factors influencing screening behavior. With this combination of findings, health care professionals could create an improved, culturally appropriate educational modality best suited for informed decision making regarding prostate screening and increase screening behavior among African American and AfroCaribbean American men (Drake et al., 2010; Friedman et al., 2009; Miller, 2014; Patel et al., 2013; Smith et al., 2012; Wolf et al., 2010).

Second, the findings have an implication for a continued implementation of the local faith-based medical partnerships, such as the CHAMP partnership, with nationally recognized partnerships, in order to help diminish knowledge gaps and health disparities (Levin, 2014). Churches, working in collaboration with other organizations in the community, have already been settings for health-related programs where African American men have improved health behaviors, and health disparities have decreased (Saunders et al., 2013). The above-mentioned national partnerships expand accessibility and help disseminate prostate screening awareness. Another concrete reason for utilizing national partnerships is Miller's (2014) finding that the health disparity of mortality rate 
from prostate cancer among African American men has decreased as participation in prostate cancer screening has increased.

A health disparity, however, still is apparent in the United States in regards to prostate cancer and ethnicity (U.S. Department of Health and Human Services, 2014). According to the U.S. Cancer Statistics Working Group (2014), the age-adjusted incidence rate for prostate cancer per 100,000 men for all races is 128.3, and Black is the ethnicity with the highest rate at 194.7 per 100,000 men. The Healthy People 2020 goal is to reduce the prostate cancer death rate from the baseline of 24.2 per 100,000 men from 2007 to the target of 21.8 per 100,000 men (U.S. Department of Health and Human Services, 2014). Educators, clinicians, and researchers working to achieve the Healthy People 2020 goal of decreasing the prostate cancer death rate should consider including faith-based medical partnerships for high-risk African American and Afro-Caribbean American men.

Third, the study findings have implications for nurses and other health professionals working to inform men of African descent about specific risks and benefits regarding prostate cancer screening. Health care professionals can take the lead in discussions to help men make an informed decision regarding screening by presenting men with culturally relevant messages and easily understood information (Miller, 2014), consistent with the American Cancer Society’s (2015) recommendation.

Nurses and other professionals at the bedside, in clinics, in medical offices, and in the community, including churches, can help address this public health problem through culturally appropriate education. Discussions with men and potentially their family members about prostate cancer screening should not only take place during a primary 
care office visit, when there are often competing priorities. Health care workers require improved tools to help patients make informed decisions regarding screening for prostate cancer, which could improve overall patient care, health outcomes, and decrease costs.

\section{Future Research}

Future studies should include the development and testing of culturally appropriate strategies and tools for nurses and other health care providers to use to promote informed decision making concerning prostate cancer screening. Theoretical constructs of the HBM, including perceived susceptibility, perceived severity, cues to action, perceived cost, and perceived benefits, require further study. To diminish the health disparity regarding prostate cancer morbidity and mortality, research focused on the influence of culture on informed decision making and readiness to participate in routine prostate cancer screening is needed.

\section{Summary}

The purpose of this study was to assess the likelihood of action for, and factors influencing, choice regarding prostate cancer screenings. It was also to discover if a relationship existed between ethnicity (African American and Afro-Caribbean American men) and knowledge of prostate health, self-efficacy (measured by men’s level of conflict in making an informed decision regarding prostate screening), perceived barriers to prostate screening, and beliefs measured by spiritual well-being. Results from this study showed there was a relationship between ethnicity (African American and AfroCaribbean American men) and the influencing factor of perceived barriers on prostate cancer screening behavior. African American participants reported structural or societal barriers to screening, yet Afro-Caribbean American participants reported personal 
barriers to screening. Also, there was a relationship between ethnicity (African American and Afro-Caribbean American men) and level of prostate health knowledge. Both ethnicities had low total prostate knowledge. In addition, there was a relationship between ethnicity (African American and Afro-Caribbean American men) and the influencing factor of beliefs measured by spiritual well-being on screening behavior. Both ethnicities had high spiritual well-being. Furthermore, there was a relationship between ethnicity (African American and Afro-Caribbean American men) and informed decision making self-efficacy measured by men’s level of conflict regarding prostate screening. Both African American and Afro-Caribbean American participants had moderate total decisional conflict.

The study findings demonstrated several barriers to screening, low knowledge levels, and moderate conflict in making a decision to participate in prostate cancer screenings, which are likely reasons for almost half of African American participants (47.9\%) and nearly a third of Afro-Caribbean American participants (29\%) not to participate in a PSA blood test (or not know if one was conducted). With decreased participation in prostate cancer screenings, mortality rates increase, contributing to the disparity of higher mortality rates from prostate cancer among these ethnic groups than among other ethnicities. Therefore, the study findings reaffirm the existence of a disparity regarding prostate cancer among the high-risk ethnic groups of African American and Afro-Caribbean American men. The results signal the necessity for further research and imply a need for more culturally appropriate education for these ethnic groups. Much has been done, but these findings indicate more still needs to be done to close the disparity and achieve the Healthy People 2020 goal of decreasing the mortality 
rate from prostate cancer. These study findings have implications for patient education, faith-based and medical partnerships, and improved health outcomes for a population at high risk for prostate cancer morbidity and mortality. 


\section{REFERENCES}

Adesanya, O. A., Shittu, L. A. J., Awobajo, F. O., Otulana, O. J., \& Adesanya, R. A. (2013). Knowledge and practice of prostate health in the work place. Journal of Infertility and Reproductive Biology, 1(2): 54-57

Allen, J. D., Othus, M. D., Hart, A., Mohllajee, A. P., Li, Y., \& Bowen, D. (2011). Do men make informed decisions about prostate cancer screening? Baseline results from the “Take the Wheel” trial. Medical Decision Making, 31(1), 108-120. doi:10.1177/0272989X10369002

American Cancer Society. (2015). Cancer facts and figures 2015. Retrieved May 2, 2015, from http://www.cancer.org/acs/groups/content/@editorial/documents/ document/acspc-044552.pdf

Anderson, B., Marshall-Lucette, S., \& Webb, P. (2013). African and Afro-Caribbean men's experiences of prostate cancer. British Journal of Nursing, 22(22), 12961307.

Andrews, C. M., Darnell, J. S., Mcbride, T. D., \& Gehlert, S. (2013). Social work and implementation of the Affordable Care Act. Health \& Social Work, 38(2), 67-71.

Archibald, C. (2011). Cultural tailoring for an Afro-Caribbean community: A naturalistic approach. Journal of Cultural Diversity, 18(4), 114-119.

Aubry, W., Lieberthal, R., Willis, A., Bagley, G., M., \& Layton, A. (2013). Budget impact model: Epigenetic assay can help avoid unnecessary repeated prostate biopsies and reduce healthcare spending. American Health \& Drug Benefits, 6(1), $1-7$.

Austin, O. (2012). Prostate-specific antigen prostate cancer screening: Answers to the critical questions. Annals of Long Term Care, 20(10), 16-21.

Basch, E., Oliver, T., Vickers, A., Thompson, I., Kantoff, P., Parnes, H., ... Nam, R. (2012). Screening for prostate cancer with prostate-specific antigen testing: American Society of Clinical Oncology provisional clinical opinion. Journal of Clinical Oncology, 30(24), 3020-3025. doi:10.1200/JCO.2012.43.3441

Bell, K., \& Kazanjian, A. (2011). PSA testing: Molecular technologies and men's experience of prostate cancer survivorship. Health, Risk \& Society, 13(2), 183198. doi:10.1080/13698575.2011.556187

Bul, M., \& Schrööder, F. H. (2011). Screening for prostate cancer -- The controversy continues, but can it be resolved?. Acta Oncologica, 504-11. doi:10.3109/0284186X.2010.522197 
Bungay, H., \& Cappello, R. (2009). 'As long as the doctors know what they are doing': Trust or ambivalence about patient information among elderly men with prostate cancer?. European Journal of Cancer Care, 18(5), 470-476. doi:10.1111/j.13652354.2008.00992.x

Burns, R., Walsh, B., Sharp, L., \& O'Neill, C. (2012). Prostate cancer screening practices in the Republic of Ireland: The determinants of uptake. Journal of Health Services Research \& Policy, 17(4), 206-211. doi:10.1258/jhsrp.2012.011105

Carter, V., Tippett, F., Anderson, D., \& Tameru, B. (2010). Increasing prostate cancer screening among African American men. Journal of Health Care for the Poor \& Underserved,21(3), 91-106. doi:10.1353/hpu.0.0366

Centers for Disease Control. (2011). Addressing the cancer burden at a glance. Retrieved August 8, 2013, from http://www.cdc.gov/chronicdisease/resources/publications/ AAG/dcpc.htm

Chou, R., Croswell, J. M., Dana, T., Bougatsos, C., Blazina, I., Fu, R., ... Lin, K. (2011). Screening for prostate cancer: A review of the evidence for the U.S. Preventive Services Task Force. Annals of Internal Medicine, 155(11):762-771.

Cobran, E. K., Wutoh, A. K., Lee, E., Odedina, F. T., Ragin, C., Aiken, W., \& Godley, P. A. (2013). Perceptions of prostate cancer fatalism and screening behavior between United States-born and Caribbean-born black males. Journal of Immigrant and Minority Health, 1-7. doi:10.1007/s10903-013-9825-5.

Cohen J. (1988). Statistical power analysis for the behavioral sciences. $2^{\text {nd }}$ ed. Lawrence Erlbaum.

Consedine, N. S. (2012). Are we worrying about the right men and are the right men feeling worried? Conscious but not unconscious prostate anxiety predicts screening among men from three ethnic groups. American Journal of Men's Health, 6(1), 37-50. doi:10.1177/1557988311415513

Cooperberg, M. R., Carroll, P. R., \& Klotz, L. (2011). Active surveillance for prostate cancer: Progress and promise. Journal of Clinical Oncology, 29(27):3669-76. doi: 10.1200/JCO.2011.34.9738.

Cross, T., Bazron, B., Dennis, K. W., \& Isaacs, M. R. (1989). Towards a culturally competent system of care: A monograph on effective services for minority children who are severely emotionally disturbed (Vol. 1). Washington, DC: Georgetown University Child Development Center, CASSP Technical Assistance Center. 
Cross, D. S., Ritter, M., \& Reding, D. J. (2012). Historical prostate cancer screening and treatment outcomes from a single institution. Clinical Medicine \& Research, 10(3), 97-105. doi:10.3121/cmr.2011.1042

Davis, J., Grant, C., Rivers, B., Rivera-Colón, V., Ramos, R., Antolino, P., ... Green, B. L. (2012). The prostate cancer screening controversy: Addressing bioethical concerns at a community health promotion event for men. Journal of Health Care for the Poor \& Underserved, 23(4), 11-14. doi:10.1353/hpu.2012.0160

Dillard, A., Couper, M., \& Zikmund-Fisher, B. (2010). Perceived risk of cancer and patient reports of participation in decisions about screening: The DECISIONS study. Medical Decision Making, 30(5S), 96S-105s. doi:10.1177/0272989X10377660

Djulbegovic, M., Beyth, R.J., Neuberger, M.M., Stoffs, T. L., Vieweg, J., Djulbegovic, B., \& Dahm, P. (2010). Screening for prostate cancer: Systematic review and meta-analysis of randomized controlled trials. BMJ. 341:c4543.

Doody, O., Slevin,E., \& Taggart, L. (2013). Focus group interviews in nursing research: Part 1. British Journal of Nursing, 22(1), 16-19

Dossey, B.M., \& Keegan, L. (2009). Holistic nursing: A handbook for practice. Jones \& Bartlett Publishers, Sudbury, MA.

Drake, B. F., Shelton, R. C., Gilligan, T., \& Allen, J. D. (2010). A church-based intervention to promote informed decision making for prostate cancer screening among African American men. Journal of the National Medical Association, 102(3), 164-171.

Eldefrawy, A., Katkoori, D., Abramowitz, M., Soloway, M., \& Manoharan, M. (2013). Active surveillance vs. treatment for low-risk prostate cancer: A cost comparison. Urologic Oncology, 31(5), 576-580. doi:10.1016/j.urolonc.2011.04.005

Emerson, J. S., Reece, M. C., Levine, R S., Hull, P. C., \& Husaini, B. A. (2009). Predictors of new screening for African American men participating in a prostate cancer educational program. Journal of Cancer Education, 24(4), 341-345. doi:10.1080/08858190902854749

Ferrante, J., Shaw, E., \& Scott, J. (2011). Factors influencing men's decisions regarding prostate cancer screening: A qualitative study. Journal of Community Health, 36(5), 839-844. doi:10.1007/s10900-011-9383-5 
Friedman, D., Corwin, S., Dominick, G., \& Rose, I. (2009). African American men's understanding and perceptions about prostate cancer: Why multiple dimensions of health literacy are important in cancer communication. Journal of Community Health, 34(5), 449-460. doi:10.1007/s10900-009-9167-3

Friedman, D., Johnson, K., Owens, O., Thomas, T., Dawkins, D., Gansauer, L., \& ... Hébert, J. (2012). Developing partnerships and recruiting dyads for a prostate cancer informed decision making program: Lessons learned from a communityacademic-clinical team. Journal of Cancer Education, 27(2), 243-249. doi:10.1007/s13187-012-0353-0

Furlow, B. (2012). Prostate cancer and radiation therapy. Radiation Therapist, 21(1), 29-56.

Gash, J., \& McIntosh, G. V. (2013). Gender matters: Health beliefs of women as a predictor of participation in prostate cancer screening among African American men. Diversity \& Equality in Health \& Care, 10(1), 23-30.

Giaimo, S. (2013). Behind the scenes of the Patient Protection and Affordable Care Act The making of a health care co-op. Journal of Health Politics, Policy \& Law, 38(3), 599-610. doi:10.1215/03616878-2079532

Glenn, B. A., Bastani, R., Maxwell, A. E., Herrmann, A. K., Swanson, K. A., Mojica, ... Chang, C. L. (2012). Prostate cancer screening among ethnically diverse firstdegree relatives of prostate cancer cases. Health Psychology, 31(5), 562-570. doi:10.1037/a0028626

Goldenberg, S. (2013). Discovering medical nemesis. Journal of Continuing Education Topics \& Issues, 15(1), 10-12.

Hevey, D., Pertl, M., Thomas, K., Maher, L., Chuinneagáin, S., \& Craig, A. (2009). The relationship between prostate cancer knowledge and beliefs and intentions to attend PSA screening among at-risk men. Patient Education \& Counseling, 74(2), 244-249. doi:10.1016/j.pec.2008.08.013

Holt, C. L., Wynn, T., Litaker, M., Southward, P., Jeames, S., \& Schulz, E. (2009). A comparison of a spiritually based and non-spiritually based educational intervention for informed decision making for prostate cancer screening among church-attending African-American men. Urologic Nursing, 29(4), 249-258.

Howard, D. H., Tangka, F. K., Guy, G. P., Ekwueme, D. U., \& Lipscomb, J. (2013). Prostate cancer screening in men ages 75 and older fell by 8 percentage points after task force recommendation. Health Affairs, 32(3), 596-602. doi:10.1377/hlthaff.2012.0555 
Ilic, D., Neuberger, M., Djulbegovic, M., \& Dahm, P. (2013). Screening for prostate cancer. Cochrane Database of Systematic Reviews, (1). doi:10.1002/14651858.CD004720.pub3

Jackson, D. D., Owens, O. L., Friedman, D. B., \& Hebert, J. R. (2014). An intergenerational approach to prostate cancer education: Findings from a pilot project in the Southeastern USA. Journal of Cancer Education, 1-8.

Janz, N. K., \& Becker, M. H. (1984). The Health Belief Model: A decade later. Health Education Quarterly,11, 1-47.

Jemal, A., Siegel, R., Xu, J., \& Ward, E. (2010). Cancer statistics, 2010. CA: A Cancer Journal for Clinicians, 60, 277-300. doi:10.3322/caac.20073

Jones, R., Steeves, R., \& Williams, I. (2010). Family and friend interactions among African-American men deciding whether or not to have a prostate cancer screening. Urologic Nursing, 30(3), 189.

Kegler, M., Hall, S., \& Kiser, M. (2010). Facilitators, challenges, and collaborative activities in faith and health partnerships to address health disparities. Health Education \& Behavior, 37(5), 665-679. doi:10.1177/1090198110363882

Kleier, J. A. (2010). Fear of and susceptibility to prostate cancer as predictors of prostate cancer screening among Haitian-American men. Urologic nursing, 30(3), 179188.

Koulikov, D., Mamber, A., Fridmans, A., Arafeh, W., \& Shenfeld, O. Z. (2012). Why I cannot find the prostate? Behind the subjectivity of rectal exam. ISRN Urology, 1-4. doi:10.5402/2012/456821

Kumar, N., Yu, D., Akinremi, T., \& Odedina, F. (2009). Comparing dietary and other lifestyle factors among immigrant Nigerian men living in the US and indigenous men from Nigeria: Potential implications for prostate cancer risk reduction. Journal of Immigrant \& Minority Health, 11(5), 391-399. doi:10.1007/s10903009-9231-1

Lee, D., Consedine, N., Gonzalez, J., \& Spencer, B. (2012). Association of healthcare barriers with prostate-specific antigen screening among African-American and Afro-Caribbean men. Urology, 80(3), 556-563. doi:10.1016/j.urology.2012.02.085

Lehto, R. H., Song, L., Stein, K. F., \& Coleman-Burns, P. (2010). Factors influencing prostate cancer screening in African American men. Western Journal of Nursing Research, 32(6), 779-793. doi:10.1177/0193945910361332 
Leininger, M. (2002). Culture care theory: A major contribution to advance transcultural nursing and practices. Journal of Transcultural Nursing, 13(3), 189-192.

Levin, J. (2014). Faith-based initiatives in health promotion: History, challenges, and current partnerships. American Journal of Health Promotion, 28(3), 139-141. doi:10.4278/ajhp.130403-CIT-149

Linder, S. K., Swank, P. R., Vernon, S. W., Mullen, P. D., Morgan, R. O., \& Volk, R. J. (2011). Validity of a low literacy version of the Decisional Conflict Scale.

Patient Education and Counseling, 85(3), 521-524.

doi:10.1016/j.pec.2010.12.012

Mariotto, A. B., Yabroff, K. R., Shao, Y., Feuer, E. J., \& Brown, M. L. (2011). Projections of the cost of cancer care in the United States: 2010-2020. Journal of the National Cancer Institute, 103(2), 117-128.

Martin, A., Lord, S., Verry, H., Stockler, M., \& Emery, J. (2013). Risk assessment to guide prostate cancer screening decisions: A cost-effectiveness analysis. Medical Journal of Australia, 198(10), 546-550.

McCormick, K., Osman, N., \& Pomerantz, M. (2010). Update on prostate cancer screening. Journal of Clinical Outcomes Management, 17(10), 470-479.

McDowell, M., Occhipinti, S., Gardiner, R., Baade, P., \& Steginga, S. (2009). A review of prostate-specific antigen screening prevalence and risk perceptions for firstdegree relatives of men with prostate cancer. European Journal of Cancer Care, 18(6), 545-555. doi:10.1111/j.1365-2354.2008.01046.x

McFall, S., Ureda, J., Byrd, T., Valdes, A., Morales, P., Scott, D., ... Chan, E. (2009). What is needed for informed decisions about prostate cancer screening: Perspectives of African-American and Hispanic men. Health Education Research, 24(2), 280-291. doi:10.1093/her/cyn018

Miller, D. B. (2014). Pre-screening age African-American males: What do they know about prostate cancer screening, knowledge, and risk perceptions?. Social Work in Health Care, 53(3), 268-288. doi:10.1080/00981389.2013.875503

Mitchell, J. (2011). Examining the influence of social ecological factors on prostate cancer screening in urban African-American men. Social Work in Health Care, 50(8), 639-655. doi:10.1080/00981389.2011.589891

Nelson, C. J., Balk, E. M., \& Roth, A. J. (2010). Distress, anxiety, depression, and emotional well-being in African-American men with prostate cancer. PsychoOncology, 19(10), 1052-1060. doi:10.1002/pon.1659 
Ng, P., Schoenfeld, E., Hennis, A., Wu, S., Leske, M. M., \& Nemesure, B. (2013). Factors influencing prostate cancer healthcare practices in Barbados, West Indies. Journal of Immigrant \& Minority Health, 15(3), 653-660. doi:10.1007/s10903012-9654-y

Nguyen, P. L., Gu, X., Lipsitz, S. R., Choueiri, T. K., Choi, W. W., Lei, Y., Hoffman, K. E. \& Hu, J. C. (2011). Cost implications of the rapid adoption of newer technologies for treating prostate cancer. Journal of Clinical Oncology, 29(12), 1517-1524. doi:10.1200/JCO.2010.31.1217

O’Connor, A. M. (1995). Validation of a decisional conflict scale. Medical Decision Making, 15(1), 25-30. doi: 10.1177/0272989X9501500105

Odedina, F. T., J., Larose-Pierre, M., Emanuel, F., Adams, A., Dagne, G. A., Pressey, S. A., \& Odedina, O. (2011). Modifiable prostate cancer risk reduction and early detection behaviors in black Men. American Journal of Health Behavior, 35(4), 470-484.

Odedina, F., Yu, D., Akinremi, T., Reams, R., Freedman, M., \& Kumar, N. (2009). Prostate cancer cognitive-behavioral factors in a West African population. Journal of Immigrant \& Minority Health, 11(4), 258-267. doi:10.1007/s10903008-9212-9

Oliver, J. S., Grindel, C. G., DeCoster, J., Ford, C. D., \& Martin, M. Y. (2011). Benefits, barriers, sources of influence, and prostate cancer screening among rural men. Public Health Nursing, 28(6), 515-522. doi:10.1111/j.1525-1446.2011.00956.x

Parchment, Y. (2004). Prostate cancer screening in African American and Caribbean males: Detriment in delay. ABNF Journal, 15(6), 116-120.

Patel, K., Kenerson, D., Wang, H., Brown, B., Pinkerton, H., Burress, M., \& ... Hargreaves, M. (2010). Factors influencing prostate cancer screening in lowincome African Americans in Tennessee. Journal of Health Care for the Poor \& Underserved, 21(1), 114-126. doi:10.1353/hpu.0.0235

Patel, K., Ukoli, F., Liu, J., Beech, D., Beard, K., Brown, B., \& ... Hargreaves, M. (2013). A community-driven intervention for prostate cancer screening in African Americans. Health Education \& Behavior, 40(1), 11-18. doi:10.1177/1090198111431275

Pedersen, V., Armes, J., \& Ream, E. (2012). Perceptions of prostate cancer in Black African and Black Caribbean men: A systematic review of the literature. PsychoOncology, 21(5), 457-468. doi:10.1002/pon.2043 
Peterman, A. H., Fitchett, G., Brady, M. J., Hernandez, L., \& Cella, D. (2002). Measuring spiritual well-being in people with cancer: The Functional Assessment of Chronic Illness Therapy-Spiritual Well-being Scale (FACIT-Sp). Annals of Behavioral Medicine, 24(1): 49-58. doi: 10.1207/S15324796ABM2401_06

Polit, D. E. (2010). Statistics and data analysis for nursing research (2nd ed.). Upper Saddle River, NJ: Pearson Education.

Radosevich, D. M., Partin, M. R., Nugent, S., Nelson, D., Flood, A. B., Holtzman, J., ... Wilt, T. J. (2004). Measuring patient knowledge of the risks and benefits of prostate cancer screening. Patient Education and Counseling, 54(2), 143-152. doi:10.1016/S0738-3991(03)00207-6

Rivera-Ramos, Z. A., \& Buki, L. P. (2011). I will no longer be a man! Manliness and prostate cancer screenings among Latino men. Psychology of Men \& Masculinity, 12(1), 13-25. doi:10.1037/a0020624

Rivers, B. M., August, E. M., Han, G., Buchanan, K. L., Rivers, D., \& Green, B. (2010). Comparative examination of prostate cancer knowledge among African Americans and Whites in Alabama. Journal of Best Practices in Health Professions Diversity: Education, Research \& Policy, 3(2), 21-44.

Rosenstock, I. M. (1966). Why people use health services. Milbank Memorial Fund Quarterly, 44(3): 94-127.

Rosenstock, I. M., Strecher, V. J., \& Becker, M. H. (1988). Social Learning Theory and the Health Belief Model. Health Education \& Behavior, 15(2): 175-183. doi:10.1177/109019818801500203

Sandblom, G., Varenhorst. E., Rosell, J., Lofman, O., \& Carlsson, P. (2011).

Randomized prostate cancer screening trial: 20 year follow-up. BMJ.342:d1539.

Saunders, D. R., Holt, C. L., Whitehead, T. L., Atkinson, N. L., Le, D., Wang, M. Q., ... \& Naslund, M. (2013). Development of the men's prostate awareness church training: Church-based workshops for African American men. Family \& community health, 36(3), 224-235. doi:10.1097/FCH.0b013e318292eb40.

Shariat, S. F., Semjonow, A., Lilja, H., Savage, C., Vickers, A. J., \& Bjartell, A. (2011). Tumor markers in prostate cancer I: Blood-based markers. Acta Oncologica, 5061-75. doi:10.3109/0284186X.2010.542174

Shavers, V., Underwood, W., \& Moser, R. (2009). Race/ethnicity, risk perception, and receipt of prostate-specific antigen testing. Journal of the National Medical Association, 101(7), 698-704. 
Shaw, S., Vivian, J., Orzech, K., Torres, C., \& Armin, J. (2012). Consistency in attitudes across cancer screenings in medically underserved minority populations. Journal of Cancer Education, 27(1), 165-171. doi:10.1007/s13187-011-0285-0

Shen, Z. (2015). Cultural Competence Models and Cultural Competence Assessment Instruments in Nursing: A literature review. Journal of Transcultural Nursing, 26(3), 308-321. doi:10.1177/1043659614524790

Skolarus, T., Zhang, Y., \& Hollenbeck, B. (2012). Understanding fragmentation of prostate cancer survivorship care: Implications for cost and quality. Cancer (0008543X), 118(11), 2837-2845. doi:10.1002/cncr.26601

Smith, R.A., Cokkinides, V., \& Brawley, O.W. (2012). Cancer screening in the United States, 2013: A review of current American Cancer Society guidelines and current issues in cancer screening. CA- A Cancer Journal for Clinicians, 62, 129142. doi:10.3322/caac. 21174

Sobo, E. J. (2009). Culture \& Meaning in Health Services Research. Walnut Creek, California: Left Coast Press, Inc.

Soydan, H., Dursun, F., Yılmaz, Ö., Okçelik, S., Ateş, F., \& Karademir, K. (2013). Clinical results of active surveillance for localized prostate cancer patients. Turkish Journal of Urology, 39(1), 1-5. doi:10.5152/tud.2013.001

Squiers, L. B., Bann, C. M., Dolina, S. E., Tzeng, J., McCormack, L., \& Kamerow, D. (2013). Prostate-specific antigen testing: Men's responses to 2012 recommendation against screening. American Journal of Preventive Medicine, 45(2), 182-189. doi:10.1016/j.amepre.2013.04.005.

Stojković, S. \& Prlić, N. (2012). Effect of faith on the acceptance of chronic disease patients. South Eastern Europe Health Sciences Journal, 2(1): 52-61.

Stranne, S. K. (2011). An oncology perspective on preventive services in the context of US healthcare reform. Oncology (08909091), 25(12), 1119-1132.

Taylor, K. L., Davis, J. L., 3rd, Turner, R. O., Johnson, L., Schwartz, M. D., Kerner, J. F., \& Leak, C. (2006). Educating African American men about the prostate cancer screening dilemma: A randomized intervention. Cancer Epidemiology Biomarkers, 15(11), 2179-2188. doi:10.1158/1055-9965.EPI-05-0417

Ugwumba, F., \& Nnabugwu, I. (2012). Digital rectal examination in the detection of prostate cancer. Perception of training received and competence amongst house officers in a resource challenged setting. Saudi Medical Journal, 33(5), 568-570. 
University of Miami (FL) Medical School, Florida Cancer Data System. (2008-2011). Prostate cancer incidence. FloridaCHARTS. Retrieved August 8, 2013, from http://www.floridacharts.com/charts/OtherIndicators/NonVitalIndDataViewer.asp x?cid $=0450$

U.S. Cancer Statistics Working Group. (2014). United States Cancer Statistics: 19992011 Incidence and Mortality Web-based Report. Atlanta (GA): Department of Health and Human Services, Centers for Disease Control and Prevention, and National Cancer Institute. Retrieved April 6, 2015, from http://apps.nccd.cdc.gov/uscs/cancersbyraceandethnicity.aspx

U.S. Department of Health and Human Services. (2014). Office of Disease Prevention and Health Promotion. Healthy People 2020. Washington, DC. Retrieved April 6, 2015, from http://www.healthypeople.gov/node/4073/data_details

van Leeuwen, P., Connolly, D., Gavin, A., Roobol, M., Black, A., Bangma, C., \& Schröder, F. (2010). Prostate cancer mortality in screen and clinically detected prostate cancer: Estimating the screening benefit. European Journal of Cancer, 46(2), 377-383. doi:10.1016/j.ejca.2009.09.008

Vickers, A.J., Roobol, M.J., \& Lilja, H. (2012). Screening for prostate cancer: Early detection or overdetection? Annual Review of Medicine, 63, 161-170. doi:10.1146/annurev-med-050710-134421

Violette, P., \& Saad, F. (2012). Chemoprevention of prostate cancer: Myths and realities. Journal of the American Board of Family Medicine, 25(1), 111-119.

Visser, A., Garssen, B., \& Vingerhoets, A. (2010). Spirituality and well-being in cancer patients: A review. Psycho-Oncology, 19(6), 565-572. doi:10.1002/pon.1626

Waltz, C. F., Strickland, O. L., \& Lenz, E. R. (2010). Measurement in nursing and health research (4th ed.). New York, NY: Springer.

Wilensky, S. E., \& Gray, E. A. (2013). Existing Medicaid beneficiaries left off the Affordable Care Act's prevention bandwagon. Health Affairs, 32(7), 1188-1195. doi:10.1377/hlthaff.2013.0224

Wolf, A.M.D., Wender, R.C., Etzioni, R.B., Thompson, I.M., D’Amico, A.V., Volk, R.J.,...Smith, R.A. (2010). American Cancer Society guideline for the early detection of prostate cancer. CA: A Cancer Journal for Clinicians, 60(2), 70-98.

Wong, Y., \& Coups, E. J. (2011). Correlates of colorectal cancer screening adherence among men who have been screened for prostate cancer. Journal of Cancer Education, 26(2), 301-307. doi:10.1007/s13187-011-0194-2 
Wray, R., McClure, S., Vijaykumar, S., Smith, C., Ivy, A., Jupka, K., \& Hess, R. (2009). Changing the conversation about prostate cancer among African Americans: Results of formative research. Ethnicity \& Health, 14(1), 27-43. 


\section{APPENDIX A}

\section{Survey: Demographic Questionnaire}

Instructions: Please enter your age on the first question, then place an $\mathrm{X}$ in the box before the answer you would like to choose.

1. How many years old are you today?

2. How do you identify your race? [] Black, [] Caucasian, [] Latino, [] Native American, [] Asian/Pacific Islander, [] Other (specify)

3. How do you identify your Ethnicity? [] Hispanic, [] African-American, [] AfroCaribbean, [] Other (specify)

4. What is your marital status? [] Single [] Widower [] Divorced [] Married [] Partnered

5. What is the highest education level you have completed?

[] Less than high school/GED [] High school/GED [] Associates degree or some college or trade school [] Bachelor's degree [] Post-baccalaureate education

6. What is your household annual income or salary?

[]$<\$ 20,000[] \$ 20,000$ to $<\$ 40,000[] \$ 40,000$ to $<\$ 60,000[] \$ 60,000$ to $<\$ 80,000$ []$>\$ 80,000$

7. What is your health insurance coverage?

[] Private insurance (Such as Blue Cross/Blue Shield)

[] Public insurance (Such as Medicaid, Medicare) [] No health insurance

8. What religion do you affiliate with?

[] Catholic [] Protestant [] Jehovah’s Witness [] Mormon/LDS [] Jewish []

Muslim [] Hindu [] Buddhist [] None [] Atheist [] Agnostic [] Other (specify)

\section{Personal and Family Health History Questionnaire}

Instructions: Please place an $\mathrm{X}$ in the box before the answer you would like to choose.

1. Is there a history of prostate cancer in your family?

[] Yes [] No [] I don't know

2. Has your doctor ever checked your prostate?

[] Yes [] No [] I don't know

3. Have you ever had a prostate-specific antigen (PSA) blood test?

[] Yes [] No [] I don't know

4. Have you ever had any of these problems? Difficulty urinating, pain on urination, frequent urination, blood in the urine.

[] Yes [] No [] I don't know 


\section{Prostate Knowledge:}

\section{Instructions:}

Mark each statement true or false with an X.

1. Most men diagnosed as having prostate cancer die of something else. [] True [] False

2. Men are more likely to die because of prostate cancer than because of heart disease.

[] True [] False

3. Prostate cancer is the most common cause of problems with urination. [] True [] False

4. Prostate cancer never causes problems with urination.

[] True [] False

5. Prostate cancer is one of the least common cancers among men.

[] True [] False

6. If you have an abnormal prostate-specific antigen (PSA) test result, your doctor may recommend that you have a prostate biopsy.

[] True [] False

7. The prostate-specific antigen (PSA) test will pick up all prostate cancers [] True [] False

8. A prostate biopsy can tell you with more certainty whether you have prostate cancer than a prostate-specific antigen (PSA) test can.

[] True [] False

9. Loss of sexual function is a common side effect of prostate cancer treatments. [] True [] False

10. Problems with urination are common side effects of prostate cancer treatments. [] True [] False

11. The prostate is an accessory organ of reproduction.

[] True [] False

12. The prostate is located in the thorax of the body.

[] True [] False 


\section{Decisional Conflict Scale:}

Instructions: Which do you prefer? Please check one.
a. Getting a prostate-specific antigen PSA test []
b. NOT getting a prostate-specific antigen PSA test []
c. Unsure []

Instructions: Considering the option you prefer, please answer the following questions:

1. Do you know which options are available to you?

[]Yes []Unsure []No

2. Do you know the benefits of each option?

[]Yes []Unsure []No

3. Do you know the risks and side effects of each option?

[]Yes []Unsure []No

4. Are you clear about which benefits matter most to you?

[]Yes []Unsure []No

5. Are you clear about which risks and side effects matter most to you?

[]Yes[]Unsure[]No

6. Do you have enough support from others to make a choice?

[]Yes []Unsure []No

7. Are you choosing without pressure from others?

[]Yes []Unsure []No

8. Do you have enough advice to make a choice?

[]Yes []Unsure []No

9. Are you clear about the best choice for you?

[]Yes []Unsure []No

10. Do you feel sure about what to choose?

[]Yes []Unsure []No 


\section{Possible Barriers to Screening:}

\section{Instructions:}

Please rate the reasons that you might not participate in a prostate screening from greatest to least with one (1) being the first or main reason you might not participate to eleven (11) being the reason you are least concerned about.

\begin{tabular}{|l|l|}
\hline Perceived Barrier: & Ranking 1-11 \\
\hline Dislike or fear of doctors & \\
\hline Can't afford it & \\
\hline Fear of finding cancer & \\
\hline No insurance & \\
\hline No transportation & \\
\hline Exam is embarrassing & \\
\hline Past problems with doctors & \\
\hline Did not think it was important & \\
\hline Doctor did not tell me I needed it & \\
\hline Personal or religious beliefs & \\
\hline Exam is uncomfortable & \\
\hline
\end{tabular}




\section{FACIT-Sp-12 (Version 4)}

Functional Assessment of Chronic Illness Therapy - Spiritual Well-Being, a modified version for non-illness

Please circle or mark one number per line to indicate your response as it applies to the past 7 days.

ADDITIONAL CONCERNS

$\begin{array}{ccccc}\text { Not } & \text { A } & \text { Some- } & \text { Quite } & \text { Very } \\ \text { at } & \text { little } & \text { what } & \text { a bit } & \text { much } \\ \text { all } & \text { bit } & & & \end{array}$

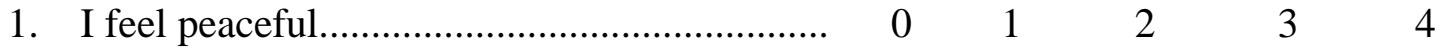

2. I have a reason for living.................................. $00 \begin{array}{lllll} & 0 & 2 & 3 & 4\end{array}$

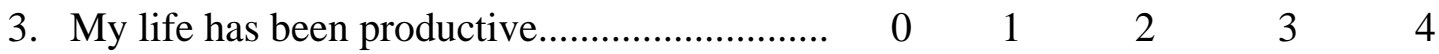

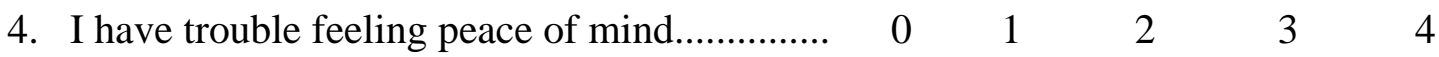

5. I feel a sense of purpose in my life.................. $0 \begin{array}{llllll} & 0 & 1 & 2 & 3 & 4\end{array}$

6. I am able to reach down deep into myself for $\begin{array}{lllllll}0 & 1 & 2 & 3 & 4\end{array}$ comfort.

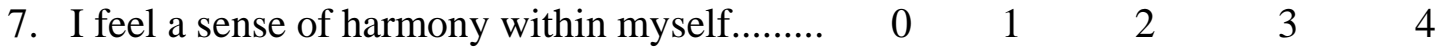

8. My life lacks meaning and purpose.................

$\begin{array}{llllllll}\text { 9. I find comfort in my faith or spiritual beliefs.. } & 0 & 1 & 2 & 3 & 4\end{array}$

10. I find strength in my faith or spiritual beliefs. $\begin{array}{lllllll}0 & 1 & 2 & 3 & 4\end{array}$

11. Difficult times have strengthened my faith or spiritual beliefs.

$\begin{array}{lllll}0 & 1 & 2 & 3 & 4\end{array}$

12. Even during difficult times, I know that things will be okay

34

English (Universal) 16 November 2007 Copyright 1987, 1997 


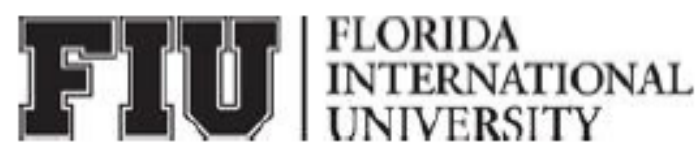

Office of Research Integrity

Research Compliance, MARC 270

\section{MEMORANDUM}

To:

Dr. Ellen Brown

$\mathrm{CC}$ :

From:

File

Date:

Maria Melendez-Vargas, MIBA, IRB Coordinator

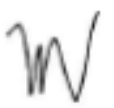

Protocol Title: $\quad$ "African American and Afro-Caribbean American Men's Prostate Health Knowledge and Beliefs"

The Health Sciences Institutional Review Board of Florida International University has approved your study for the use of human subjects via the Expedited Review process. Your study was found to be in compliance with this institution's Federal Wide Assurance (00000060).

IRB Protocol Approval \#: IRB-14-0370

IRB Approval Date: $\quad 12 / 04 / 14$

TOPAZ Reference \#: 103018

IRB Expiration Date: 12/04/15

As a requirement of IRB Approval you are required to:

1) Submit an IRB Amendment Form for all proposed additions or changes in the procedures involving human subjects. All additions and changes must be reviewed and approved by the IRB prior to implementation.

2) Promptly submit an IRB Event Report Form for every serious or unusual or unanticipated adverse event, problems with the rights or welfare of the human subjects, and/or deviations from the approved protocol.

3) Utilize copies of the date stamped consent document(s) for obtaining consent from subjects (unless waived by the IRB). Signed consent documents must be retained for at least three years after the completion of the study.

4) Receive annual review and re-approval of your study prior to your IRB expiration date. Submit the IRB Renewal Form at least 30 days in advance of the study's expiration date.

5) Submit an IRB Project Completion Report Form when the study is finished or discontinued.

Special Conditions: N/A

For further information, you may visit the IRB website at http://research fiu.edu/irb. 
APPENDIX C

\begin{tabular}{|c|c|c|}
\hline $\begin{array}{l}\text { Table C1 } \\
\text { Major Concepts and Empirical Indicators }\end{array}$ & & \\
\hline Variable & Instrument & Reference \\
\hline $\begin{array}{l}\text { Independent Variable(IV) } 1= \\
\text { How many years old are you today? }\end{array}$ & $\begin{array}{l}\text { Demographic } \\
\text { Questionnaire }\end{array}$ & $\begin{array}{l}\text { Parchment, } \\
2004 .\end{array}$ \\
\hline $\begin{array}{l}\text { Independent Variable(IV) 2= } \\
\text { How do you identify your race? [] Black, [] } \\
\text { Caucasian, [] Latino, [] Native American, [] } \\
\text { Asian/Pacific Islander, [] Other (specify) }\end{array}$ & $\begin{array}{l}\text { Demographic } \\
\text { Questionnaire }\end{array}$ & $\begin{array}{l}\text { Parchment, } \\
2004 .\end{array}$ \\
\hline $\begin{array}{l}\text { Independent Variable(IV) 3= } \\
\text { How do you identify your Ethnicity? [] } \\
\text { Hispanic, [] African-American, [] Afro- } \\
\text { Caribbean, [] Other (specify) }\end{array}$ & $\begin{array}{l}\text { Demographic } \\
\text { Questionnaire }\end{array}$ & $\begin{array}{l}\text { Parchment, } \\
2004 .\end{array}$ \\
\hline $\begin{array}{l}\text { Independent Variable(IV) } 4= \\
\text { What is your marital status? [] Single } \\
\text { [] Widower [] Divorced [] Married [] Partnered }\end{array}$ & $\begin{array}{l}\text { Demographic } \\
\text { Questionnaire }\end{array}$ & $\begin{array}{l}\text { Parchment, } \\
2004 .\end{array}$ \\
\hline $\begin{array}{l}\text { Independent Variable(IV) } 5= \\
\text { What is the highest education level you have } \\
\text { completed? } \\
\text { []Less than high school/GED []High } \\
\text { school/GED []Associates degree or some } \\
\text { college or trade school []Bachelor's } \\
\text { degree[]Post-baccalaureate education }\end{array}$ & $\begin{array}{l}\text { Demographic } \\
\text { Questionnaire }\end{array}$ & $\begin{array}{l}\text { Parchment, } \\
2004 .\end{array}$ \\
\hline $\begin{array}{l}\text { Independent Variable(IV) } 6= \\
\text { What is your household annual income or } \\
\text { salary? } \\
{[]<\$ 20,000[] \$ 20,000 \text { to }<\$ 40,000[] \$ 40,000 \text { to }<} \\
\$ 60,000[] \$ 60,000 \text { to }<\$ 80,000[]>\$ 80,000\end{array}$ & $\begin{array}{l}\text { Demographic } \\
\text { Questionnaire }\end{array}$ & $\begin{array}{l}\text { Parchment, } \\
2004 .\end{array}$ \\
\hline $\begin{array}{l}\text { Independent Variable(IV) } 7= \\
\text { What is your health insurance coverage? } \\
\text { [] Private insurance (Such as Blue Cross/Blue } \\
\text { Shield) [] Public insurance (Such as Medicaid, } \\
\text { Medicare) [] No health insurance }\end{array}$ & $\begin{array}{l}\text { Demographic } \\
\text { Questionnaire }\end{array}$ & $\begin{array}{l}\text { Parchment, } \\
2004 .\end{array}$ \\
\hline $\begin{array}{l}\text { Independent Variable(IV) 8= } \\
\text { What religion do you affiliate with? } \\
\text { [] Catholic [] Protestant [] Jehovah's Witness [] } \\
\text { Mormon/LDS [] Jewish [] Muslim [] } \\
\text { Hindu [] Buddhist [] None [] Atheist [] } \\
\text { Agnostic [] Other (specify) }\end{array}$ & $\begin{array}{l}\text { Demographic } \\
\text { Questionnaire }\end{array}$ & $\begin{array}{l}\text { Parchment, } \\
2004 .\end{array}$ \\
\hline $\begin{array}{l}\text { Independent Variable(IV) 9= } \\
\text { Is there a history of prostate cancer in your } \\
\text { family? } \\
\text { [] Yes [] No [] I don't know }\end{array}$ & $\begin{array}{l}\text { Personal and } \\
\text { Family Health } \\
\text { History } \\
\text { Questionnaire }\end{array}$ & $\begin{array}{l}\text { Parchment, } \\
2004 .\end{array}$ \\
\hline
\end{tabular}




\begin{tabular}{|c|c|c|}
\hline $\begin{array}{l}\text { Independent Variable(IV) } 10= \\
\text { Has your doctor ever check your prostate? } \\
\text { [] Yes [] No [] I don't know }\end{array}$ & $\begin{array}{l}\text { Personal and } \\
\text { Family Health } \\
\text { History } \\
\text { Questionnaire }\end{array}$ & $\begin{array}{l}\text { Parchment, } \\
2004 .\end{array}$ \\
\hline $\begin{array}{l}\text { Independent Variable(IV) } 11= \\
\text { Have you ever had a prostate-specific antigen } \\
\text { (PSA) blood test? } \\
\text { [] Yes [] No [] I don't know }\end{array}$ & $\begin{array}{l}\text { Personal and } \\
\text { Family Health } \\
\text { History } \\
\text { Questionnaire }\end{array}$ & $\begin{array}{l}\text { Parchment, } \\
2004 .\end{array}$ \\
\hline $\begin{array}{l}\text { Independent Variable(IV) } 12= \\
\text { Have you ever had any of these problems? } \\
\text { Difficulty urinating, pain on urination, frequent } \\
\text { urination, blood in the urine. } \\
\text { [] Yes [] No [] I don't know }\end{array}$ & $\begin{array}{l}\text { Personal and } \\
\text { Family Health } \\
\text { History } \\
\text { Questionnaire }\end{array}$ & $\begin{array}{l}\text { Parchment, } \\
2004 .\end{array}$ \\
\hline $\begin{array}{l}\text { Dependent Variable(DV) } 1= \\
\text { Most men diagnosed as having } \\
\text { prostate cancer die of something else } \\
\text { [] True [] False }\end{array}$ & $\begin{array}{l}\text { Prostate Cancer } \\
\text { Screening } \\
\text { Education } \\
\text { (PROCASE) } \\
\text { Knowledge Index }\end{array}$ & $\begin{array}{l}\text { Radosevich et } \\
\text { al., 2004. }\end{array}$ \\
\hline $\begin{array}{l}\text { Dependent Variable(DV) } 2= \\
\text { Men are more likely to die because of } \\
\text { prostate cancer than because of heart } \\
\text { disease } \\
\text { [] True [] False }\end{array}$ & $\begin{array}{l}\text { PROCASE } \\
\text { Knowledge Index }\end{array}$ & $\begin{array}{l}\text { Radosevich et } \\
\text { al., } 2004 \text {. }\end{array}$ \\
\hline $\begin{array}{l}\text { Dependent Variable(DV) } 3= \\
\text { Prostate cancer is the most common } \\
\text { cause of problems with urination } \\
\text { [] True [] False }\end{array}$ & $\begin{array}{l}\text { PROCASE } \\
\text { Knowledge Index }\end{array}$ & $\begin{array}{l}\text { Radosevich et } \\
\text { al., 2004. }\end{array}$ \\
\hline $\begin{array}{l}\text { Dependent Variable(DV) } 4= \\
\text { Prostate cancer never causes problems } \\
\text { with urination } \\
\text { [] True [] False }\end{array}$ & $\begin{array}{l}\text { PROCASE } \\
\text { Knowledge Index }\end{array}$ & $\begin{array}{l}\text { Radosevich et } \\
\text { al., 2004. }\end{array}$ \\
\hline $\begin{array}{l}\text { Dependent Variable(DV) } 5= \\
\text { Prostate cancer is one of the least } \\
\text { common cancers among men } \\
\text { [] True [] False }\end{array}$ & $\begin{array}{l}\text { PROCASE } \\
\text { Knowledge Index }\end{array}$ & $\begin{array}{l}\text { Radosevich et } \\
\text { al., } 2004 \text {. }\end{array}$ \\
\hline $\begin{array}{l}\text { Dependent Variable(DV) 6= } \\
\text { If you have an abnormal prostate } \\
\text { specific antigen (PSA) test result, } \\
\text { your doctor may recommend that you } \\
\text { have a prostate biopsy } \\
\text { [] True [] False }\end{array}$ & $\begin{array}{l}\text { PROCASE } \\
\text { Knowledge Index }\end{array}$ & $\begin{array}{l}\text { Radosevich et } \\
\text { al., 2004. }\end{array}$ \\
\hline $\begin{array}{l}\text { Dependent Variable(DV) } 7= \\
\text { The prostate-specific antigen (PSA) } \\
\text { test will pick up all prostate cancers } \\
\text { [] True [] False }\end{array}$ & $\begin{array}{l}\text { PROCASE } \\
\text { Knowledge Index }\end{array}$ & $\begin{array}{l}\text { Radosevich et } \\
\text { al., 2004. }\end{array}$ \\
\hline
\end{tabular}




\begin{tabular}{|c|c|c|}
\hline $\begin{array}{l}\text { Dependent Variable(DV) } 8= \\
\text { A prostate biopsy can tell you with } \\
\text { more certainty whether you have } \\
\text { prostate cancer than a prostate-specific antigen } \\
\text { (PSA) test can } \\
\text { [] True [] False }\end{array}$ & $\begin{array}{l}\text { PROCASE } \\
\text { Knowledge Index }\end{array}$ & $\begin{array}{l}\text { Radosevich et } \\
\text { al., } 2004 \text {. }\end{array}$ \\
\hline $\begin{array}{l}\text { Dependent Variable(DV) } 9= \\
\text { Loss of sexual function is a common } \\
\text { side effect of prostate cancer } \\
\text { treatments } \\
\text { [] True [] False }\end{array}$ & $\begin{array}{l}\text { PROCASE } \\
\text { Knowledge Index }\end{array}$ & $\begin{array}{l}\text { Radosevich et } \\
\text { al., } 2004 \text {. }\end{array}$ \\
\hline $\begin{array}{l}\text { Dependent Variable(DV) } 10= \\
\text { Problems with urination are common } \\
\text { side effects of prostate cancer } \\
\text { treatments } \\
\text { [] True [] False }\end{array}$ & $\begin{array}{l}\text { PROCASE } \\
\text { Knowledge Index }\end{array}$ & $\begin{array}{l}\text { Radosevich et } \\
\text { al., } 2004 \text {. }\end{array}$ \\
\hline $\begin{array}{l}\text { Dependent Variable(DV) } 11= \\
\text { The prostate is an accessory organ of } \\
\text { reproduction } \\
\text { [] True [] False }\end{array}$ & $\begin{array}{l}\text { Knowledge } \\
\text { and Practice } \\
\text { of Prostate } \\
\text { Health } \\
\text { Questionnaire }\end{array}$ & $\begin{array}{l}\text { Adesanya, } \\
\text { Shittu, } \\
\text { Awobajo, } \\
\text { Otulana, \& } \\
\text { Adesanya, } 2013 \\
\end{array}$ \\
\hline $\begin{array}{l}\text { Dependent Variable(DV) } 12= \\
\text { The prostate is located in the thorax of the body } \\
\text { [] True [] False }\end{array}$ & $\begin{array}{l}\text { Knowledge } \\
\text { and Practice } \\
\text { of Prostate } \\
\text { Health } \\
\text { Questionnaire }\end{array}$ & $\begin{array}{l}\text { Adesanya et al., } \\
2013 .\end{array}$ \\
\hline $\begin{array}{l}\text { Dependent Variable(DV) 13= } \\
\text { Do you know which options are available to } \\
\text { you? } \\
\text { [] Yes [] Unsure [] No }\end{array}$ & $\begin{array}{l}\text { Low Literacy } \\
\text { Decisional Conflict } \\
\text { Scale }\end{array}$ & $\begin{array}{l}\text { O’Connor, } \\
1995 .\end{array}$ \\
\hline $\begin{array}{l}\text { Dependent Variable(DV) 14= } \\
\text { Do you know the benefits of each option? } \\
\text { [] Yes [] Unsure [] No }\end{array}$ & $\begin{array}{l}\text { Low Literacy } \\
\text { Decisional Conflict } \\
\text { Scale }\end{array}$ & $\begin{array}{l}\text { O’Connor, } \\
1995 .\end{array}$ \\
\hline $\begin{array}{l}\text { Dependent Variable(DV) 15= } \\
\text { Do you know the risks and side effects of each } \\
\text { option? } \\
\text { [] Yes [] Unsure [] No }\end{array}$ & $\begin{array}{l}\text { Low Literacy } \\
\text { Decisional Conflict } \\
\text { Scale }\end{array}$ & $\begin{array}{l}\text { O’Connor, } \\
1995 .\end{array}$ \\
\hline $\begin{array}{l}\text { Dependent Variable(DV) } 16= \\
\text { Are you clear about which benefits matter most } \\
\text { to you? } \\
\text { [] Yes [] Unsure [] No }\end{array}$ & $\begin{array}{l}\text { Low Literacy } \\
\text { Decisional Conflict } \\
\text { Scale }\end{array}$ & $\begin{array}{l}\text { O’Connor, } \\
1995 .\end{array}$ \\
\hline $\begin{array}{l}\text { Dependent Variable(DV) } 17= \\
\text { Are you clear about which risks and side effects } \\
\text { matter most to you? } \\
\text { [] Yes [] Unsure [] No }\end{array}$ & $\begin{array}{l}\text { Low Literacy } \\
\text { Decisional Conflict } \\
\text { Scale }\end{array}$ & $\begin{array}{l}\text { O’Connor, } \\
1995 .\end{array}$ \\
\hline
\end{tabular}




\begin{tabular}{|c|c|c|}
\hline $\begin{array}{l}\text { Dependent Variable(DV) } 18= \\
\text { Do you have enough support from others to } \\
\text { make a choice? } \\
\text { [] Yes [] Unsure [] No }\end{array}$ & $\begin{array}{l}\text { Low Literacy } \\
\text { Decisional Conflict } \\
\text { Scale }\end{array}$ & $\begin{array}{l}\text { O’Connor, } \\
1995 .\end{array}$ \\
\hline $\begin{array}{l}\text { Dependent Variable(DV) } 19= \\
\text { Are you choosing without pressure from others? } \\
\text { [] Yes [] Unsure [] No }\end{array}$ & $\begin{array}{l}\text { Low Literacy } \\
\text { Decisional Conflict } \\
\text { Scale }\end{array}$ & $\begin{array}{l}\text { O’Connor, } \\
1995 .\end{array}$ \\
\hline $\begin{array}{l}\text { Dependent Variable(DV) } 20= \\
\text { Do you have enough advice to make a choice? } \\
\text { [] Yes [] Unsure [] No }\end{array}$ & $\begin{array}{l}\text { Low Literacy } \\
\text { Decisional Conflict } \\
\text { Scale }\end{array}$ & $\begin{array}{l}\text { O’Connor, } \\
1995 .\end{array}$ \\
\hline $\begin{array}{l}\text { Dependent Variable(DV) 21= } \\
\text { Are you clear about the best choice for you? } \\
\text { [] Yes [] Unsure [] No }\end{array}$ & $\begin{array}{l}\text { Low Literacy } \\
\text { Decisional Conflict } \\
\text { Scale }\end{array}$ & $\begin{array}{l}\text { O’Connor, } \\
1995 .\end{array}$ \\
\hline $\begin{array}{l}\text { Dependent Variable(DV) } 22= \\
\text { Do you feel sure about what to choose? } \\
\text { [] Yes [] Unsure [] No }\end{array}$ & $\begin{array}{l}\text { Low Literacy } \\
\text { Decisional Conflict } \\
\text { Scale }\end{array}$ & $\begin{array}{l}\text { O’Connor, } \\
1995 .\end{array}$ \\
\hline $\begin{array}{l}\text { Dependent Variable(DV) } 23= \\
\text { Please rate the reasons that you might not } \\
\text { participate in a prostate screening from greatest } \\
\text { (being number one (1)) to least (being number } \\
\text { eleven (11)): } \\
\text { [] Dislike or fear of doctors } \\
\text { [] Can't afford it } \\
\text { [] Fear of finding cancer } \\
\text { [] No insurance } \\
\text { [] No transportation } \\
\text { [] Exam is embarrassing } \\
\text { [] Past problems with doctors } \\
\text { [] Did not think it was important } \\
\text { [] Doctor did not tell me I needed it } \\
\text { [] Personal or religious beliefs } \\
\text { [] Exam is uncomfortable }\end{array}$ & $\begin{array}{l}\text { Perceived Barriers } \\
\text { of the Cancer } \\
\text { Awareness Survey }\end{array}$ & $\begin{array}{l}\text { Parchment, } \\
2004 .\end{array}$ \\
\hline $\begin{array}{l}\text { Dependent Variable(DV) 24= } \\
\text { I feel peaceful } \\
\text { []Not at all [] A little bit []Somewhat } \\
\text { [] Quite a bit [] Very much }\end{array}$ & $\begin{array}{l}\text { Functional } \\
\text { Assessment of } \\
\text { Chronic Illness } \\
\text { Therapy-Spiritual } \\
\text { Well-Being, a } \\
\text { modified version } \\
\text { for non-illness } \\
\text { (FACIT-Sp Non- } \\
\text { Illness) (peace } \\
\text { subscale) }\end{array}$ & $\begin{array}{l}\text { Visser, Garssen, } \\
\text { \& Vingerhoets } \\
(2010)\end{array}$ \\
\hline $\begin{array}{l}\text { Dependent Variable(DV) } 25= \\
\text { I have a reason for living } \\
\text { []Not at all [] A little bit []Somewhat }\end{array}$ & $\begin{array}{l}\text { FACIT-Sp non- } \\
\text { illness (meaning } \\
\text { subscale) }\end{array}$ & $\begin{array}{l}\text { Visser et al., } \\
2010 .\end{array}$ \\
\hline
\end{tabular}




\begin{tabular}{|c|c|c|}
\hline [] Quite a bit [] Very much & & \\
\hline $\begin{array}{l}\text { Dependent Variable(DV) } 26= \\
\text { My life has been productive } \\
\text { []Not at all [] A little bit []Somewhat } \\
\text { [] Quite a bit [] Very much }\end{array}$ & $\begin{array}{l}\text { FACIT-Sp non- } \\
\text { illness (meaning } \\
\text { subscale) }\end{array}$ & $\begin{array}{l}\text { Visser et al., } \\
2010 .\end{array}$ \\
\hline $\begin{array}{l}\text { Dependent Variable(DV) } 27= \\
\text { I have trouble feeling peace of mind } \\
\text { []Not at all [] A little bit []Somewhat } \\
\text { [] Quite a bit [] Very much }\end{array}$ & $\begin{array}{l}\text { FACIT-Sp non- } \\
\text { illness (peace } \\
\text { subscale) }\end{array}$ & $\begin{array}{l}\text { Visser et al., } \\
2010 .\end{array}$ \\
\hline $\begin{array}{l}\text { Dependent Variable(DV) } 28= \\
\text { I feel a sense of purpose in my life } \\
\text { []Not at all [] A little bit []Somewhat } \\
\text { [] Quite a bit [] Very much }\end{array}$ & $\begin{array}{l}\text { FACIT-Sp non- } \\
\text { illness (meaning } \\
\text { subscale) }\end{array}$ & $\begin{array}{l}\text { Visser et al., } \\
2010 .\end{array}$ \\
\hline $\begin{array}{l}\text { Dependent Variable(DV) } 29= \\
\text { I am able to reach down deep into myself for } \\
\text { comfort } \\
\text { []Not at all [] A little bit []Somewhat } \\
\text { [] Quite a bit [] Very much }\end{array}$ & $\begin{array}{l}\text { FACIT-Sp non- } \\
\text { illness (peace } \\
\text { subscale) }\end{array}$ & $\begin{array}{l}\text { Visser et al., } \\
2010 .\end{array}$ \\
\hline $\begin{array}{l}\text { Dependent Variable(DV) } 30= \\
\text { I feel a sense of harmony within myself } \\
\text { []Not at all [] A little bit []Somewhat } \\
\text { [] Quite a bit [] Very much }\end{array}$ & $\begin{array}{l}\text { FACIT-Sp non- } \\
\text { illness (peace } \\
\text { subscale) }\end{array}$ & $\begin{array}{l}\text { Visser et al., } \\
2010 .\end{array}$ \\
\hline $\begin{array}{l}\text { Dependent Variable(DV) 31= } \\
\text { My life lacks meaning and purpose } \\
\text { []Not at all [] A little bit []Somewhat } \\
\text { [] Quite a bit [] Very much }\end{array}$ & $\begin{array}{l}\text { FACIT-Sp non- } \\
\text { illness (meaning } \\
\text { subscale) }\end{array}$ & $\begin{array}{l}\text { Visser et al., } \\
2010 .\end{array}$ \\
\hline $\begin{array}{l}\text { Dependent Variable(DV) } 32= \\
\text { I find comfort in my faith or spiritual beliefs } \\
\text { []Not at all [] A little bit []Somewhat } \\
\text { [] Quite a bit [] Very much }\end{array}$ & $\begin{array}{l}\text { FACIT-Sp non- } \\
\text { illness (faith } \\
\text { subscale) }\end{array}$ & $\begin{array}{l}\text { Visser et al., } \\
2010 .\end{array}$ \\
\hline $\begin{array}{l}\text { Dependent Variable(DV) 33= } \\
\text { I find strength in my faith or spiritual beliefs } \\
\text { []Not at all [] A little bit []Somewhat } \\
\text { [] Quite a bit [] Very much }\end{array}$ & $\begin{array}{l}\text { FACIT-Sp non- } \\
\text { illness (faith } \\
\text { subscale) }\end{array}$ & $\begin{array}{l}\text { Visser et al., } \\
2010 .\end{array}$ \\
\hline $\begin{array}{l}\text { Dependent Variable(DV) } 34= \\
\text { Difficult times have strengthened my faith or } \\
\text { spiritual beliefs } \\
\text { []Not at all [] A little bit []Somewhat } \\
\text { [] Quite a bit [] Very much }\end{array}$ & $\begin{array}{l}\text { FACIT-Sp non- } \\
\text { illness (faith } \\
\text { subscale) }\end{array}$ & $\begin{array}{l}\text { Visser et al., } \\
2010 .\end{array}$ \\
\hline $\begin{array}{l}\text { Dependent Variable(DV) 35= } \\
\text { Even during difficult times, I know that things } \\
\text { will be okay } \\
\text { []Not at all [] A little bit []Somewhat } \\
\text { [] Quite a bit [] Very much }\end{array}$ & $\begin{array}{l}\text { FACIT-Sp non- } \\
\text { illness (faith } \\
\text { subscale) }\end{array}$ & $\begin{array}{l}\text { Visser et al., } \\
2010 .\end{array}$ \\
\hline
\end{tabular}


VITA

\section{CORA YOOSE}

2007

2008-2009

2009-2011

2011-2014

2012-2014

2013

2014-present

2014-present

2014-present
Bachelor of Science in Nursing Brigham Young University

Provo, UT

Registered Nurse/Program Manager

Area Health Education Center (AHEC)

Marathon, FL

Registered Nurse

Cyberknife Institute

Radiation/Oncology Department

Mercy Hospital

Miami, FL

Registered Nurse

Orthopedic and Rehabilitation Floors

Mercy Hospital

Miami, FL

Assistant Nurse Manager

Rehabilitation Floor

Mercy Hospital

Miami, FL

Master of Science in Nursing

Family Nurse Practitioner

Florida International University

Miami, FL

Adjunct Laboratory Faculty

Barry University

Miami Shores, FL

Adjunct Faculty

Florida International University

Miami, FL

Full-time Faculty

Fortis College

Cutler Bay, FL 


\section{PUBLICATIONS AND PRESENTATIONS}

Yoose, C. (2011). Book Review: Teaching cultural competence in nursing and health care (2nd ed.). ABNF Journal, 22(4), 96-97.

Yoose, C. Spiritually Based Educational Intervention on Prostate Cancer for African-American Men. South Florida Nursing Research Conference. Coral Gables, Florida, October, 2011 (poster presentation). 